is generally correlated with environment, is mostly detected in low-density environments (e.g., Sarzi et al. 2007; Shapiro et al. 2010, and references therein), and is organized into disk-like and ring configurations.

There is no clear spectral distinction between the post-mainsequence or low-mass stellar populations that can produce a large amount of UV emission. Blue post-main-sequence stellar populations, such as blue horizontal branch stars (BHBs) with extreme temperatures and low-mass stellar envelopes, are promising candidates. At higher temperatures $\left(T_{\text {eff }} \gtrsim 14,000 \mathrm{~K}\right)$ BHBs are called extended (sometimes "extreme") HBs (EHBs; Code \& Welch 1979; Burstein et al. 1988; Greggio \& Renzini 1990; Ferguson \& Davidsen 1993; Brown et al. 1995, 2003; Atlee et al. 2009; Conroy \& Gunn 2010; Yi et al. 2011). Dorman et al. (1993) found that the driving factor for increasing the temperatures of BHBs is the mass of the envelope in the zeroage $\mathrm{HB}$ phase, where a smaller envelope allows for higher temperatures; higher metallicities will result in a bimodal distribution of temperatures for envelope masses of $0.05-0.15 M_{\odot}$. The morphology of the HB in the color-magnitude diagram is critically determined by the Reimers mass-loss parameter $(\eta$; e.g., Percival \& Salaris 2011), which describes the efficiency of the mass loss of the outer envelope in the HB phase. The complexities of the BHB phase are not easily isolated with broad-band photometry or low-resolution spectroscopy. It is difficult to distinguish the BHB stars from the upper main-sequence stars using line strengths. These complications are in addition to the familiar age-metallicity degeneracy (Lee et al. 2000; Trager et al. 2005; Conroy \& Gunn 2010).

A metal-rich star would produce UVX through significant mass loss in the red giant branch phase: a resulting lower mass HB (asymptotic giant branch, AGB manqué) star could sustain $T_{\text {eff }} \sim 20,000 \mathrm{~K}$ for $\sim 10 \mathrm{Myr}$ (e.g., O’Connell 1999). Lowmetallicity stars may also play a part since the oldest populations are thought to be metal poor; however, models suggest that the UVX is not present until $>15 \mathrm{Gyr}$ for $Z \lesssim 0.5 Z_{\odot}$ (Yi et al. 1998). Observations show a lack of a UV upturn in metalpoor galactic clusters as compared with giant ellipticals (Yi et al. 1998 and references therein). Additionally, models that include UV upturn show that solar, or higher metallicities allow UVX at younger ages ( $<5 \mathrm{Gyr})$ compared with metal-poor populations (>10 Gyr; see Figure 9 in Yi et al. 1998). However, this is strongly dependent on the mass-loss efficiency. It would require a significant amount ( $>20 \%$ of HB stars) to produce the observed FUV in galaxies (O'Connell 1999), making the ages and lifetime of EHB phases an important factor. In globular clusters, up to $30 \%$ of the HB stars appear to be in an EHB phase (Kaluzny \& Udalski 1992; Liebert et al. 1994).

Binary stars provide a promising alternative since their environments can host the conditions for rapid loss of the hydrogen envelope, forming hot helium-burning stars. There are no metallicity requirements for producing UVX in binaries. Observed candidates for such binaries include hot subdwarf (sdBs) stars in globular clusters (see review by Heber 2009). Han et al. (2007) model the UVX produced by binary sdBs in which stellar populations as young as $1 \mathrm{Gyr}$ can reach these extreme temperatures and reproduce the colors seen for ETGs.

Knowledge about how massive ellipticals evolve to their present state is important for understanding galaxy evolution. The theories need to integrate many factors including the morphologies and age-metallicity differences observed between more and less massive ETGs. Mergers play an important role in explaining these factors. For example, dissipational (gas-rich) mergers would seed new star formation, while a dissipationless (gas-poor) merger would add mass via combining older stellar populations. An older stellar population in the outer halo may also be explained by outside-in cessation, where the star formation ceases first in the disk (e.g., Pipino \& Matteucci 2004; Pipino et al. 2006). In an inside-out cessation, a core forms through very rapid starbursts at high redshifts, and through major multi-stage growth at later epochs, the outer regions form via minor mergers and accretion of hot gas from the immediate environment (e.g., Daddi et al. 2005; Nelson et al. 2012; Saracco et al. 2012).

The cores of nearby ETGs (within a half-light or effective radius) have been studied in much greater detail than the extended regions. The most recent and comprehensive study, the SAURON survey (de Zeeuw et al. 2002), uses the SAURON integral-field spectrograph to survey 72 nearby galaxies; 48 are ETGs. Among the sample, a kinematic separation is identified that separates the ETGs into subpopulations of slow and fast rotators based on their projected angular momentum (Emsellem et al. 2007). The slow rotators tend to be less compact and composed of older stellar populations ( $>8 \mathrm{Gyr}$; McDermid et al. 2006). Additionally, a slight trend with Mg II and FUV - NUV color is found for the slow rotators. The fast rotators have ages of $<5$ Gyr. Shapiro et al. (2010) use mid-infrared (mid-IR) colors with Spitzer to trace low-level star formation through polycyclic aromatic hydrocarbon (PAH) emission in the sample. Most significantly, they find that the slow rotators have no sign of PAH emission and are quiescent over an $\sim 8$ Gyr period.

In summary, much literature has been written to address the origin of UV emission in ETGs, and how they may have evolved into their present form. Resolved spectral analysis on ETGs has mostly focused on scales slightly larger than the effective radius, because of practicality. For example, Jeong et al. (2012) use resolved spectroscopy to test the Burstein relation on galaxies with extended UV. However, the Mg lines used are within the effective radii. Another example is the work of Carter et al. (2011), who compare the UV and near-IR colors for the total flux and central regions of ETGs to determine if the FUV excess is related to velocity dispersion, metallicity, or abundance ratio. However, their radial profiles do not extend beyond $\sim 30^{\prime \prime}$ from the galactocentric radius of each galaxy. These studies and similar ones do not use stellar population modeling to study the central and outer regions simultaneously to the spatial extent and resolution that is possible with photometry. In this paper, we conduct such an analysis as the first step in an expanded, and detailed, series of papers.

We use mid-IR imaging from the Wide-field Infrared Survey Explorer (WISE) to explore the UV to mid-IR color-space of ETGs with UVX. The two main objectives in our study are to (1) determine a how photometry between the UV and mid-IR can be used to interpret the likely stellar populations contributing to the UV emission and (2) use radial information from these results to interpret how these galaxies may have evolved. We do this over the total flux of the galaxies in our sample to analyze the color differences between the inner and outer regions. WISE provides the all-sky capabilities for a complete study. In Section 2, we discuss our selection and the detailed extended photometry. In Section 3 we describe the stellar population synthesis models and parameters used for our analysis. We explore the color gradients, spatial distribution, and possible source of the UVX in our sample in Section 4. We conclude with a discussion on the physical origin and the possible formation scenarios that may explain our results in 
Section 5. Where necessary, we adopt a $\Lambda$ CDM cosmology, $\left(\Omega_{\mathrm{m}}, \Omega_{\Lambda}, H_{0}\right)=\left(0.27,0.73,71 \mathrm{~km} \mathrm{~s}^{-1} \mathrm{Mpc}^{-1}\right)$.

\section{SAMPLE SELECTION}

We selected ETGs from the GALEX-Ultraviolet Atlas of Nearby Galaxies (Gil de Paz et al. 2007). This atlas includes galaxies from the GALEX Nearby Galaxy Survey and galaxies within that field and other fields with similar or greater depth. They select galaxies based on optical parameters from de Vaucouleurs et al. (1991) with $\mu_{\mathrm{B}}=25 \mathrm{mag} \operatorname{arcsec}^{-2}$ isophote diameters larger than 1'; they also use the de Vaucouleurs et al. (1991) galaxy types to label the morphologies. The atlas contains a total of 1034 galaxies where 893 have both FUV and NUV detections. For our sample, we selected by morphological type E or S0, resulting in a sample of 125 galaxies all at $z<0.06$. We then applied three further criteria. First, since we selected the sources during the execution of the WISE survey, not all sources had full-depth WISE data at the time the analysis was performed. We therefore removed the sources without full-depth WISE data. This amounted to a random cull of the parent sample. Second, we removed sources that did not have coverage in both Galaxy Evolution Explorer (GALEX) channels. Third, we removed sources that lay near bright foreground objects, or had obvious artifacts in the data. This resulted in a final sample of 49 reliable objects at a mean redshift of 0.02 . Since the selections we applied to the Gil de Paz et al. sample are effectively random, our final sample should be representative of the local UV- and mid-IR-bright ETG population as a whole.

\subsection{Data}

We cross-matched our sample with three catalogs: the Sloan Digital Sky Survey (DR7 SDSS; York et al. 2000, $u, g, r, i$, and $z$ filters) the Two Micron All Sky Survey (2MASS, $K_{\mathrm{s}}$-band; Skrutskie et al. 2006), and WISE (3.4, 4.6, 12, and $22 \mu \mathrm{m}$ filters; Wright et al. 2010). This provides photometry in up to 14 bands, over $0.15-22 \mu \mathrm{m}$. The WISE point-spread functions for co-added images in 3.4, 4.6, 12, and $22 \mu \mathrm{m}$ are 8.3, 9.1, 9.5, and 16.8 arcsec, respectively. The sample comprises $25 \mathrm{~S} 0$ and 24 E galaxies with a mean $3.4 \mu \mathrm{m}$ radius of $R_{\text {tot }}=55 \mathrm{kpc}$.

To obtain integrated magnitudes, we proceed as follows. For 2MASS, we obtain data from the extended source catalog (2MASS XSC; Jarrett et al. 2003). For WISE we obtain magnitudes from the extended source catalog project (WISE XS), which is described in Jarrett et al. (2013) and briefly here. For each galaxy, the total flux is integrated over an elliptical surface determined by the convergence of the surface brightness profile and the background. The background was estimated by an outer annulus, the same minor/major-axis ratio and position angle ( $b / a$ and P.A.) as the elliptical aperture. Each image was visually inspected to remove foreground stars.

For GALEX and SDSS, we follow the methods in Neill et al. (2011). In the same method as Neill et al. (2011), we obtain archival GALEX images to coadd for the deepest possible image. We used both GALEX imaging filters (Martin et al. 2005): FUV $\left(\lambda_{\text {eff }}=1539 \AA\right)$ and NUV $\left(\lambda_{\text {eff }}=2316 \AA\right)$. For the SDSS data, Neill et al. $(2009,2011)$ found the SDSS catalog data to be inaccurate due to the galaxies extending over multiple strips and consequently having been broken into subregions. The authors developed a coadd and mosaic technique to obtain the proper integrated fluxes over multiple frames. We use these fluxes, and further correct for Galactic extinction using the dust maps of Schlegel et al. (1998).
Table 1

Early-type Galaxy Sample Properties

\begin{tabular}{|c|c|c|c|c|c|c|c|}
\hline Object & R.A. & Decl. & $b / a^{\mathrm{a}}$ & $\begin{array}{l}R_{\mathrm{tot}}^{\mathrm{b}} \\
(\mathrm{kpc})\end{array}$ & $\begin{array}{c}R_{\mathrm{h}}^{\mathrm{b}} \\
(\mathrm{kpc})\end{array}$ & $\begin{array}{c}\mathrm{c} z^{\mathrm{c}} \\
\left(\mathrm{km} \mathrm{s}^{-1}\right)\end{array}$ & Type $^{d}$ \\
\hline NGC 155 & $00: 34: 40.0$ & $-10: 45: 58.7$ & 0.87 & 75 & 8.1 & 6210 & S0 \\
\hline NGC 163 & $00: 35: 59.7$ & $-10: 07: 17.5$ & 0.93 & 60 & 7.7 & 5982 & $\mathrm{E}$ \\
\hline IC 1698 & $01: 25: 22.0$ & 14:50:19.7 & 0.41 & 27 & 8.7 & 6503 & S0 \\
\hline IC 1700 & $01: 25: 24.5$ & $14: 51: 52.8$ & 0.81 & 58 & 8.6 & 6356 & $\mathrm{E}$ \\
\hline UGC 1040 & $01: 27: 35.8$ & $-01: 06: 18.3$ & 0.25 & 32 & 6.3 & 4489 & S0-a \\
\hline NGC 1047 & 02:40:32.7 & $-08: 08: 51.4$ & 0.55 & 13 & 1.8 & 1340 & S0-a \\
\hline NGC 1052 & $02: 41: 04.8$ & $-08: 15: 21.0$ & 0.71 & 32 & 1.9 & 1510 & E4 \\
\hline NGC 1060 & $02: 43: 15.0$ & $32: 25: 30.0$ & 0.74 & 86 & 7.2 & 5190 & S0 \\
\hline NGC 1066 & $02: 43: 49.9$ & $32: 28: 29.7$ & 0.71 & 62 & 5.8 & 4346 & $\mathrm{E}$ \\
\hline NGC 1361 & $03: 34: 17.7$ & $-06: 15: 54.0$ & 0.68 & 50 & 6.0 & 5272 & $\mathrm{E}$ \\
\hline UGC 4188 & 08:03:24.0 & 41:54:53.3 & 0.50 & 108 & 13.9 & & S0 \\
\hline UGC 4551 & 08:44:06.0 & $49: 47: 37.6$ & 0.31 & 13 & 2.4 & 1749 & S0 \\
\hline NGC 2675 & $08: 52: 04.9$ & $53: 37: 02.3$ & 0.69 & 103 & 12.3 & 9231 & $\mathrm{E}$ \\
\hline IC 522 & $08: 54: 34.9$ & $57: 10: 00.7$ & 0.81 & 42 & 7.1 & 5079 & S0 \\
\hline NGC 2693 & $08: 56: 59.2$ & $51: 20: 50.7$ & 0.69 & 76 & 6.6 & 4942 & E3 \\
\hline UGC 4702 & $08: 58: 51.2$ & $38: 48: 34.4$ & 0.93 & 76 & 11.1 & 8443 & S0 \\
\hline NGC 2768 & $09: 11: 37.4$ & $60: 02: 14.2$ & 0.40 & 31 & 1.7 & 1373 & $\mathrm{E}$ \\
\hline NGC 3265 & $10: 31: 06.7$ & $28: 47: 47.7$ & 0.73 & 8 & 1.7 & 1319 & So \\
\hline NGC 3377 & $10: 47: 42.3$ & 13:59:09.0 & 0.47 & 16 & 1.0 & 665 & E5 \\
\hline UGC 5928 & $10: 49: 47.1$ & $51: 53: 39.0$ & 0.98 & 59 & 9.8 & 7393 & S0 \\
\hline NGC 3522 & $11: 06: 40.4$ & 20:05:07.6 & 0.53 & 12 & 1.1 & 1221 & $\mathrm{E}$ \\
\hline NGC 3539 & 11:09:08.8 & $28: 40: 19.9$ & 0.33 & 74 & 12.8 & 9707 & S0-a \\
\hline UGC 6435 & $11: 25: 35.0$ & $00: 46: 05.5$ & 0.78 & 99 & 10.1 & 7604 & S0 \\
\hline UGC 6683 & $11: 43: 16.2$ & $19: 44: 55.4$ & 0.35 & 39 & 9.2 & 7542 & $\mathrm{~S} 0-\mathrm{a}$ \\
\hline NGC 3844 & $11: 44: 00.8$ & $20: 01: 45.6$ & 0.26 & 46 & 8.9 & & S0-a \\
\hline NGC 4187 & $12: 13: 29.3$ & $50: 44: 29.0$ & & 117 & 12.1 & & $\mathrm{E}$ \\
\hline NGC 4458 & $12: 28: 57.6$ & $13: 14: 30.9$ & 0.93 & 7 & 0.8 & 635 & E0 \\
\hline NGC 4478 & $12: 30: 17.4$ & $12: 19: 42.4$ & 0.81 & 15 & 1.8 & 1349 & E2 \\
\hline M87 & $12: 30: 49.4$ & $12: 23: 28.0$ & 0.93 & 52 & 1.8 & 1307 & $\mathrm{E}$ \\
\hline IC 3457 & $12: 31: 51.4$ & $12: 39: 25.0$ & 0.68 & 17 & 2.1 & 1297 & E3 \\
\hline NGC 4787 & $12: 54: 05.5$ & 27:04:07.0 & 0.32 & 52 & 10.4 & 7585 & S0-a \\
\hline NGC 4797 & $12: 54: 55.1$ & $27: 24: 45.7$ & 0.74 & 84 & 10.1 & & S0 \\
\hline NGC 4827 & $12: 56: 43.5$ & $27: 10: 43.2$ & 0.79 & 61 & 9.9 & 7630 & $\mathrm{E}$ \\
\hline NGC 4952 & $13: 04: 58.3$ & 29:07:19.7 & 0.60 & 58 & 8.2 & 5968 & $\mathrm{E}$ \\
\hline NGC 5004 & $13: 11: 01.5$ & 29:38:12.1 & 0.72 & 57 & 9.3 & 7044 & S0 \\
\hline NGC 5173 & $13: 28: 25.3$ & $46: 35: 29.5$ & 0.96 & 22 & 3.2 & 2419 & E0 \\
\hline NGC 5329 & $13: 52: 10.0$ & 02:19:30.4 & 1.00 & 102 & 9.7 & 7109 & $\mathrm{E}$ \\
\hline UGC 8986 & $14: 04: 15.8$ & $04: 06: 44.7$ & 0.98 & 9 & 1.8 & 1232 & S0 \\
\hline NGC 5576 & $14: 21: 03.6$ & $03: 16: 15.4$ & 0.66 & 30 & 2.0 & 1487 & E3 \\
\hline NGC 5638 & $14: 29: 40.3$ & 03:14:00.5 & 0.87 & 20 & 2.3 & 1676 & $\mathrm{E}$ \\
\hline IC 1024 & $14: 31: 27.1$ & 03:00:32.2 & 0.43 & 15 & 2.0 & 1454 & S0 \\
\hline IC 1071 & $14: 54: 12.4$ & 04:45:00.9 & 0.63 & 107 & 10.2 & 8302 & S0 \\
\hline NGC 5813 & $15: 01: 11.2$ & $01: 42: 07.2$ & 0.66 & 51 & 2.6 & 1972 & $\mathrm{E}$ \\
\hline UGC 10261 & $16: 11: 04.0$ & $52: 27: 02.1$ & 0.68 & 235 & 25.0 & 19018 & S0 \\
\hline Mrk 501 & $16: 53: 52.2$ & $39: 45: 36.6$ & 0.78 & 107 & 13.3 & 10092 & $\mathrm{E}$ \\
\hline NGC 6364 & $17: 24: 27.3$ & $29: 23: 25.4$ & 0.78 & 74 & 9.3 & 6874 & So \\
\hline UGC 10935 & $17: 38: 43.2$ & $57: 14: 21.6$ & 0.51 & 49 & 12.7 & 8801 & S0 \\
\hline NGC 7317 & $22: 35: 51.8$ & $33: 56: 41.8$ & 0.95 & 56 & 8.2 & 6599 & $\mathrm{E} 4$ \\
\hline NGC 7432 & 22:58:01.9 & 13:08:04.4 & 0.59 & 134 & 9.8 & 7615 & $\mathrm{E}$ \\
\hline
\end{tabular}

Notes.

a Ellipticities at the total major and minor axes are based on the extended photometry measurements in the SDSS $r$-band.

b Total and half-light radii come from the WISE $3.4 \mu \mathrm{m}$ photometry.

${ }^{c}$ Radial velocities were obtained from the NASA Extragalactic Database archive (http://ned.ipac.caltech.edu/).

d The Hubble type, according to the Gil de Paz et al. (2007) UV catalog.

Table 1 includes some of the extended photometry from this analysis: ellipticity ( $r$-band) and WISE $3.4 \mu \mathrm{m}$ total and halflight radii. The galaxies span a wide range of total radii from $7<R_{\text {tot }}<235 \mathrm{kpc}$ with half-light radii from $0.8-25 \mathrm{kpc}$. The smallest half-light radius $\left(16^{\prime \prime}\right)$ is bigger than the point-spread 
function for the WISE images. The largest galaxies have total radii exceeding $100 \mathrm{kpc}$ : UGC $10261\left(R_{\mathrm{tot}} / R_{\mathrm{h}}=235 / 25 \mathrm{kpc}\right)$, $\operatorname{NGC} 7432\left(R_{\mathrm{tot}} / R_{\mathrm{h}}=134 / 9.8 \mathrm{kpc}\right)$, and NGC $4187\left(R_{\mathrm{tot}} / R_{\mathrm{h}}=\right.$ $117 / 12.1 \mathrm{kpc})$. Approximately $30 \%$ of the sample is highly elliptical $(a / b<0.6)$. The sample has a mean radial velocity, $\mathrm{cz}$, of $5396 \mathrm{~km} \mathrm{~s}^{-1}$.

Five of the ETGs in our sample were observed as part of the SAURON two-dimensional kinematic survey: NGC 2768, NGC 3377, NGC 4458, M87, and NGC 5813. They have been classified as slow rotators, indicative of a quiescent stellar population (McDermid et al. 2006). Bureau et al. (2011) find that the quiescent, slow rotators have blue FUV - NUV colors $(0.5 \lesssim$ FUV - NUV $\lesssim 2)$. We note that the SAURON survey analysis is limited to a narrow spatial range within the half-light radius.

\section{ANALYSIS}

In this present work, we consider three possible sources for the observed UVX in ETGs: (1) a significant population of BHB $/$ EHB stars $\left(t_{\text {age }}>2 \mathrm{Gyr}\right.$ and $\left.T_{\text {eff }}>14,000 \mathrm{~K}\right)$, where EHBs are the combined BHB phase and higher $T_{\text {eff }}$, (2) a substantial population of hot young stars from a recent smallscale starburst $\left(t_{\text {age }} \lesssim 500 \mathrm{Myr}\right.$ and $1<Z<1.5 Z_{\odot}, T_{\text {eff }}>$ $10,000 \mathrm{~K}$ ), and (3) a significant population of very hot, postmain-sequence metal-poor stars $\left(t_{\text {age }} \gtrsim 9 \mathrm{Gyr}\right.$ and $\left.Z<0.5 Z_{\odot}\right)$. We seek to distinguish between these possibilities by comparing their predicted UV/optical/mid-IR colors.

We use the Flexible Stellar Population Synthesis models (FSPS; Conroy et al. 2009; Conroy \& Gunn 2010) to create composite stellar population (CSP) models. The main advantage of the Conroy et al. (2009) models is the inclusion of a BHB phase, and the flexible treatments of the post-AGB and HB evolutionary phases. This flexibility allows one to choose the age at which the BHB phase is initiated and the percentage of thermally pulsating asymptotic giant branch (TP-AGB) stars that enter that phase. In essence, adding the BHB/EHB fractions to the CSPs drives the colors blueward after $t_{\text {age }}=2 \mathrm{Gyr}$. We combine the FSPS models with the Padova isochrones (Marigo \& Girardi 2007; Marigo et al. 2008) and the BaSEL stellar libraries (Lejeune et al. 1997, 1998), which provide mass, age, and metallicity ranges of $0.15 \leqslant M \leqslant 100 M_{\odot}$, $10^{6.6}<t<10^{10.2} \mathrm{yr}$, and $0.005<Z / Z_{\odot}<1.5\left(Z_{\odot} \equiv 0.019\right)$, respectively.

For this analysis, we consider the $\mathrm{BHB} / \mathrm{EHB}$ phase and young star sources of UVX. We also include a comparison between subsolar, solar, and super-solar metallicities. We limit the metallicities to $0.25,1$, and $1.5 Z_{\odot}$; ages between $30 \mathrm{Myr}$ and $14.1 \mathrm{Gyr}$ at $\Delta \log (t / \mathrm{yr})=0.025$ intervals; apply a Charlot $\&$ Fall (2000) dust parameter of 0.3; and use the Chabrier (2003) initial mass function (IMF).

We assume that stars on the $\mathrm{HB}$ are allowed to become BHB $/$ EHB at ages $>2$ Gyr, based on Yi et al. (1998). They show a UV slope increase for $1-1.5 Z_{\odot}$ populations during the post-AGB epoch, beginning at ages of $\gtrsim 2.5 \mathrm{Gyr}$. We take three different fractions of stars on the HB that are in the BHB (or EHB) phase: $0 \%, 25 \%$, and $50 \%$. Note that, for all populations, we have the same total number of stars on the HB; the fraction refers to the division between "ordinary" HB stars and blue (B) or extended (E) HB stars.

The division of stars in the $\mathrm{B} / \mathrm{E}$ phase between BHBs and EHBs is determined by temperature, with higher temperatures meaning more $\mathrm{E}$ (extended) than $\mathrm{B}$ (blue). The increased temperature is assumed to be due to small stellar envelope masses $\left(<0.05 M_{\odot}\right)$, where the radiation is allowed to escape freely. This analysis integrates temperature increases of log $\Delta_{T}\left(\log T_{\text {eff }}\right)$ from $0.0,0.2$, and 0.4 dex, which is a measure of the change from $T_{\text {eff }}$ (e.g., Marigo et al. 2008; Conroy et al. 2009). The parameter $\Delta_{T}$ is the shift in $T_{\text {eff }}$ for a TP-AGB phase, and is included in the FSPS models based on Marigo et al. (2008). We chose those values based on the ranges presented for a super-solar metallicity isochrone in Figure 1 of Marigo et al. (2008) to compare extreme boundaries in our parameter range. Finally, we apply three different $e$-folding times to vary the star formation history (SFH) and stellar mass with age $(\tau=0.2,0.6$ and $1 \mathrm{Gyr}$ ).

We use the FSPS models to generate tracks for color analysis. Specifically, we wish to determine which combination of $\mathrm{UV} /$ optical/mid-IR color separates populations in as many of the following variables as possible: age $\left(t_{\text {age }}\right)$, metallicity $(Z)$, change in temperature $\left(\Delta_{T}\right)$, SFH $(\tau)$, and BHB/EHB fraction. The plots in Figure 1 provide a sample of the explored colorspace/parameter combinations. These are an example of three color-space combinations with $Z=1 Z_{\odot}$. The top, middle, and bottom rows are NUV $-r / g-r, \mathrm{FUV}-\mathrm{NUV} / \mathrm{NUV}-[3.4]$, and FUV - NUV/NUV $-r$, respectively. The panels in each row display the same templates, but the lines are color-coded differently to emphasize how the parameters are evolving with BHB fraction (left), $e$-folding time $\tau$ in Gyr (middle), and the increase in effective temperature $\Delta_{T}\left(\log \left(T_{\text {eff }}\right)\right)$ at HB onset (right). For example, the left panels in each row show when the BHB fraction takes effect, when the lines have a slower rise in NUV $-r$ (top), or when they shift sharply down (blueward) in FUV - NUV (middle and bottom); the middle columns highlight the track separations for a given $\tau$ and therefore trace the SFH and the right panel does the same for temperature change. A more detailed description of the effects of different stellar population parameters on the UV, optical, and mid-IR colors is included in the Appendix.

In principle, one can do this with a UV, or UV-optical slope in the $y$-axis, and an optical-near-IR, or optical-mid-IR slope in the $x$-axis. The ultimate goal is to see which colorspaces work best, particularly extending the color-spaces into the WISE channels (from the near-IR or red optical) to determine what combination provides improved discriminatory power. We investigated several color-spaces, and determined that a UV plus WISE combination does significantly better than the UV plus optical (see the Appendix); we address this choice briefly in Section 4.2.

\section{RESULTS}

\subsection{Radial Color Gradients}

To study radial differences between UV, optical and mid-IR, we show color gradients for NUV $-r$ and NUV $-[3.4]$ in Figure 2. In Table 2, we list the NUV - [3.4] and NUV $-r$ colors at 0.3 and $0.7 R / R_{90}$ fractional radii for each ETG $\left(R_{90}\right.$ is the radius at $90 \%$ of the total flux in the $r$-band). For almost all galaxies in our sample, there is a trend toward bluer colors with increasing radius. To investigate this trend, in Figure 3 we plot the histograms of NUV $-r$ and NUV $-[3.4]$ colors at the inner $\left(R_{\text {in }}, R\right.$ within $50 \%$ flux, white $)$ and outer $\left(R_{\text {out }}, R\right.$ between $50 \%-90 \%$ flux) radii. The flux-fraction is based on the total $r$-band flux for each galaxy. Both histograms show a statistically significant color separation between peaks of approximately $1 \mathrm{mag}$. The averages are: NUV $-r=5.7 \pm 0.2$ and $4.9 \pm 0.1$ for $R_{\text {in }}$ and $R_{\text {out }}$, respectively, and NUV - [3.4] $=6.1 \pm 0.2$ 

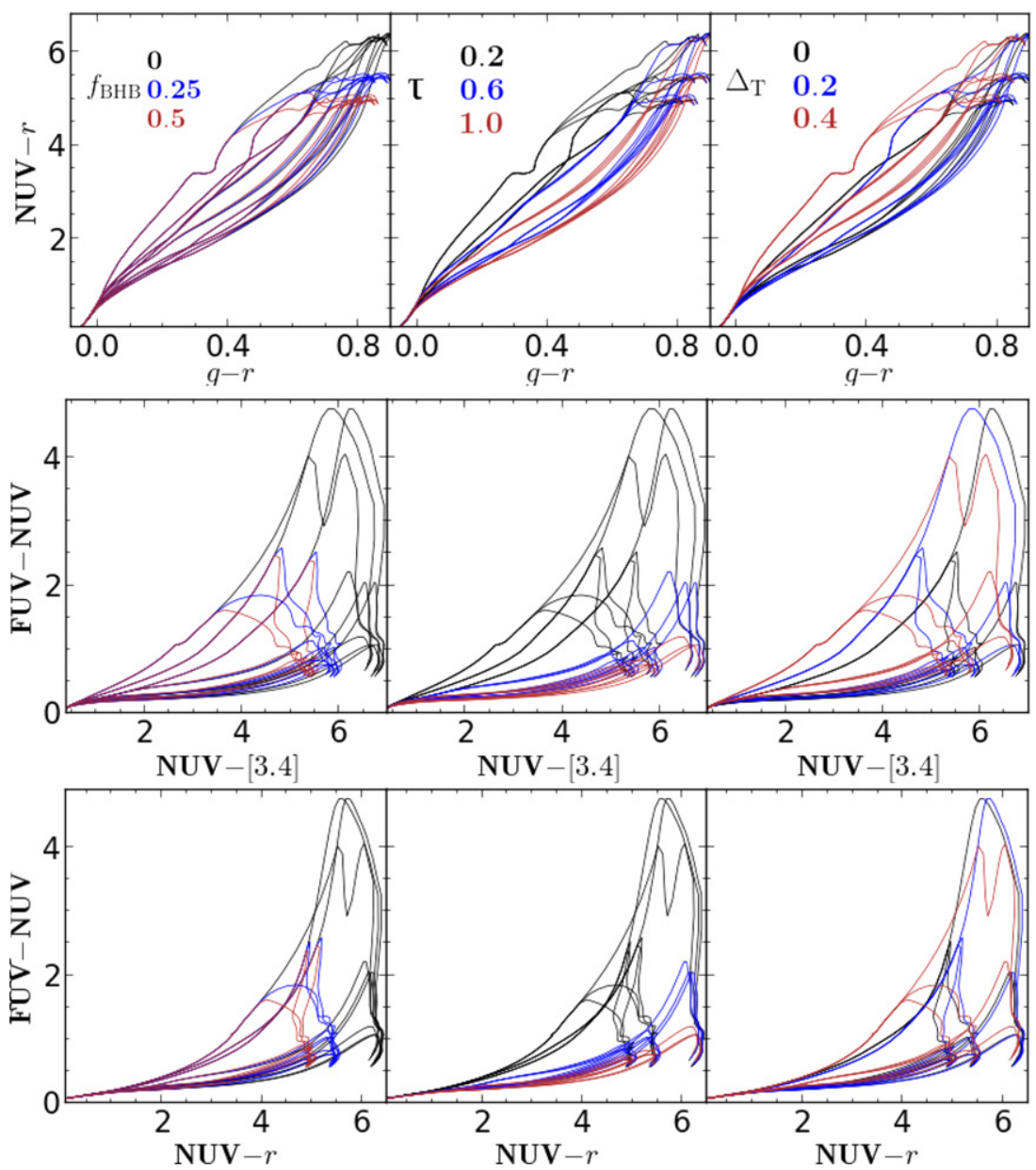

Figure 1. Flexible Stellar Population Synthesis (FSPS; Conroy et al. 2009), composite stellar population (CSP) templates depicting the evolution of color-space within an age range of 0.03-14.1 Gyr. The three rows show NUV $-r / g-r$ (top), FUV - NUV/NUV - [3.4] (middle), and FUV - NUV/NUV $-r$ (bottom) all with $Z=1 Z_{\odot}$. The left/middle/right panels in each row show the same templates/color-color values. We color-code the lines differently (left to right, as labeled in the top row) to emphasize how the parameters are evolving with BHB fraction, $e$-folding time $\tau$ in Gyr, and the increase in effective temperature $\Delta_{T}$ (log $\left.\left(T_{\text {eff }}\right)\right)$ at $\mathrm{HB}$ onset.

and $5.1 \pm 0.2$ for $R_{\text {in }}$ and $R_{\text {out }}$, respectively. The bluest galaxy, Mrk 501, is an outlier in the sample and a known blazar.

We also highlight subpopulations in the histograms based on ellipticity and morphological type (Figure 3). Morphological type does not appear to be a significant factor in the different colors in the inner and outer regions since their colors distribute similarly. Highly elliptical systems $(b / a<0.6)$ have mean $R_{\text {in }}$ colors that are preferentially reddened by $\sim 2 \%$ for NUV $-r$ and by $\sim 3 \%$ for NUV - [3.4], which is insignificant for our results. We address how dust may affect the UV to mid-IR colors in the following section.

\subsection{Comparisons to FSPS Templates}

To determine the origins of the color differences, we compare the sample UV, optical, and mid-IR colors of the $R_{\text {in }}$ and $R_{\text {out }}$ regions to the CSP models derived from the FSPS templates. In Section 3, we described Figure 1 to show how changes in the parameters can dramatically alter the paths of the FSPS tracks in a given color plot. From this figure and the Appendix, it can be seen that the FUV, NUV, $r$, and [3.4] plots provide a broader range in color-space with which to discriminate the dominating parameters for a given color region than the other optical color combinations. For example, $g-r$ and $g-i$ show a color range of approximately a magnitude (Figure 1 , and Figures 10 and 11), while NUV $-r$ and NUV - [3.4] extend over 6 mag (Figure 1, and Figures 12 and 13). Therefore, we focus on these colors in the following analysis.

In Figure 4, we show the FSPS tracks for NUV $-r$, NUV [3.4], and FUV - NUV versus age $\left(0.2<t_{\text {age }}<14.1\right.$ Gyr $)$. The left, middle, and right panels for each color-age plot are different temperature boosts given to the TP-AGB phase: $\Delta_{T}=0,0.2$, and 0.4 as labeled in the top row. The most significant effects on color are metallicity (different colors) and SFH $(\tau)$. As is commonly known, higher metallicities redden the color and a smaller $\tau$ results in a more rapid color increase. A stellar population with $Z>Z_{\odot}$ and short $e$-folding time $\sim 0.2$ can become very red by $t_{\text {age }}=2$ Gyr.

The ETG sample colors are shown in gray and orange shaded regions that span the $R_{\text {in }}$ and $R_{\text {out }}$ values, respectively. The darker horizontal gray and orange lines mark the mean NUV $-r$, NUV - [3.4], and FUV - NUV values for the corresponding color shaded area. As stated previously, the inner radii have a significantly redder color than the outer radii in NUV $-r$ and NUV - [3.4]. However, this reverses for FUV - NUV, where the inner color is, on average, bluer by a small margin ( $\sim 0.1 \mathrm{mag})$. As the stellar populations age, the possible physical drivers behind the observed colors without assuming priors, quickly become degenerate. As a generalized interpretation of the color differences in Figure 4, the following results are based on the 

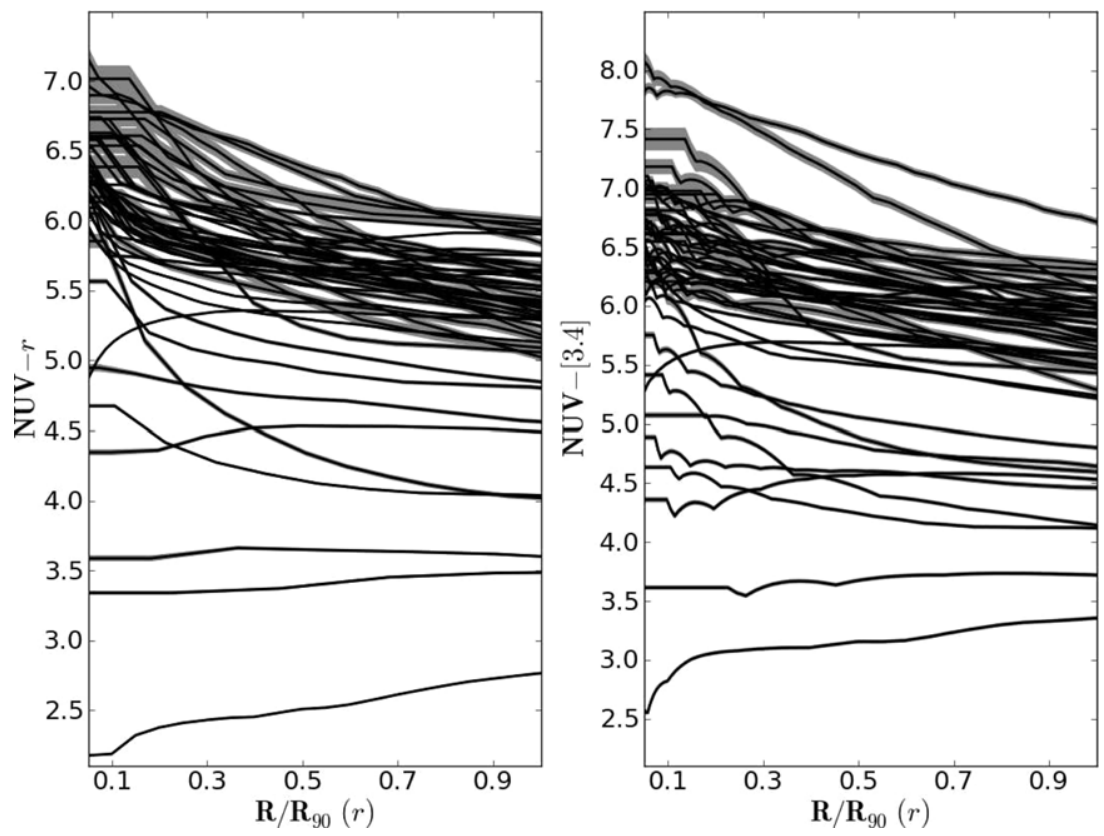

Figure 2. Color as a function of fractional $r$-band radii: NUV $-r$ (left) and NUV - [3.4] (right). The fractional radius is taken at $R / R_{90}$, where $R_{90}$ is the radius at $90 \%$ of the total flux in the $r$-band. There is a distinct trend toward bluer colors outward of the half-light radius. The gray shading is the estimated error. Table 2 lists NUV $-[3.4]$ and NUV $-r$ values at 0.3 and $0.7 R / R_{90}$.

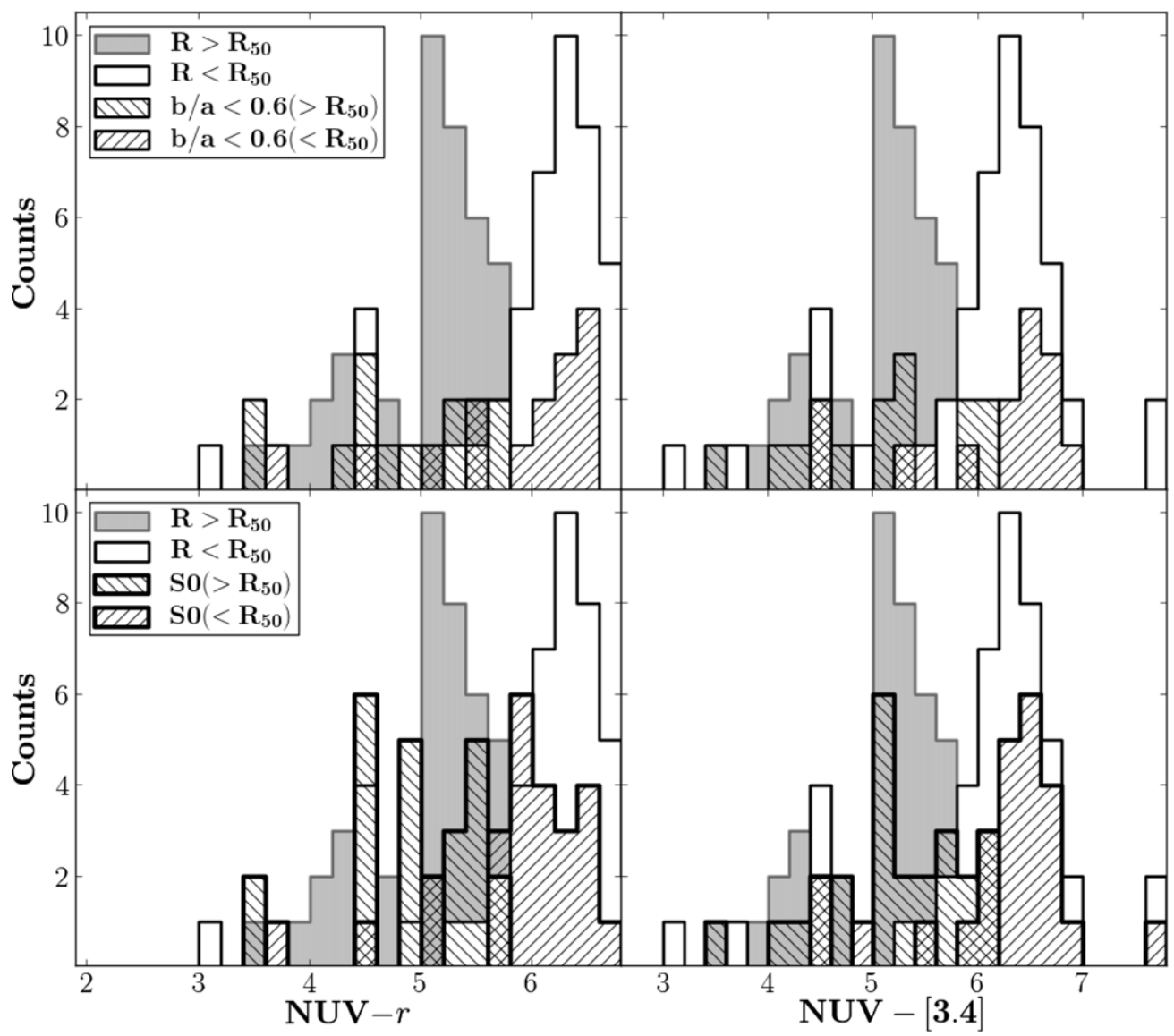

Figure 3. Distributions of NUV $-r$ and NUV - [3.4] colors at the inner $\left(R_{\text {in }}, R\right.$ within 50\% flux, white) and outer ( $R_{\text {out }}, R$ between 50\%-90\% flux, gray) radii. The hatches in the top panels accent a subpopulation of highly elliptical galaxies $(b / a<0.6)$; the bottom highlight S0 galaxies. There is a distinct color separation between the inner and outer colors, agreeing with the trend seen in Figure 2. This separation occurs at NUV - [3.4] $\gtrsim 6$, which is a strong color cut for galaxies with low BHB fraction and $1-1.5 Z_{\odot}$ (see Section 3 and the Appendix; Figure 13).

mean colors (gray and orange horizontal lines) for $R_{\text {in }}$ and $R_{\text {out }}$ at ages 2, 5, and $10 \mathrm{Gyr}$ (vertical white lines).

We summarize the parameter combinations consistent with the $R_{\text {in }}$ and $R_{\text {out }}$ mean NUV $-r$, NUV - [3.4], and FUV - NUV colors in Table 3. $R_{\text {in }}$ tends toward higher metallicities than $R_{\text {out }}$ for $t_{\text {age }} \lesssim 5$ Gyr. For both regions, $\tau$ values are limited to short $e$-folding times $(0.2 \mathrm{Gyr})$ at $t_{\mathrm{age}} \sim 2 \mathrm{Gyr}$; the $e$-folding time is degenerate at 5 and $10 \mathrm{Gyr}$. The BHB fraction is impossible 
Table 2

UV-Optical and UV-Mid-IR Colors at Specific Radii

\begin{tabular}{|c|c|c|c|c|}
\hline Object & $\begin{array}{c}\mathrm{NUV}-[3.4]^{\mathrm{a}} \\
R / R_{90}=0.3\end{array}$ & $\begin{array}{c}\mathrm{NUV}-[3.4]^{\mathrm{b}} \\
R / R_{90}=0.7\end{array}$ & $\begin{array}{c}\mathrm{NUV}-r^{\mathrm{a}} \\
R / R_{90}=0.3\end{array}$ & $\begin{array}{c}\mathrm{NUV}-r^{\mathrm{b}} \\
R / R_{90}=0.7\end{array}$ \\
\hline NGC 155 & $6.23 \pm 0.03$ & $5.81 \pm 0.02$ & $6.09 \pm 0.03$ & $5.58 \pm 0.02$ \\
\hline NGC 163 & $6.37 \pm 0.02$ & $6.15 \pm 0.02$ & $6.05 \pm 0.02$ & $5.77 \pm 0.01$ \\
\hline IC 1698 & $6.00 \pm 0.04$ & $5.14 \pm 0.02$ & $5.61 \pm 0.04$ & $4.64 \pm 0.02$ \\
\hline IC 1700 & $6.53 \pm 0.03$ & $6.07 \pm 0.02$ & $6.17 \pm 0.03$ & $5.72 \pm 0.02$ \\
\hline UGC 1040 & $6.85 \pm 0.05$ & $6.52 \pm 0.03$ & $6.76 \pm 0.05$ & $6.28 \pm 0.03$ \\
\hline NGC 1047 & $4.33 \pm 0.01$ & $4.55 \pm 0.01$ & $4.38 \pm 0.01$ & $4.53 \pm 0.01$ \\
\hline NGC 1052 & $6.42 \pm 0.01$ & $6.10 \pm 0.01$ & $5.89 \pm 0.01$ & $5.67 \pm 0.01$ \\
\hline NGC 1060 & $7.79 \pm 0.03$ & $7.31 \pm 0.03$ & $6.88 \pm 0.03$ & $6.43 \pm 0.03$ \\
\hline NGC 1066 & $7.84 \pm 0.06$ & $7.13 \pm 0.05$ & $6.91 \pm 0.06$ & $6.25 \pm 0.04$ \\
\hline NGC 1361 & $6.16 \pm 0.03$ & $5.80 \pm 0.03$ & $5.93 \pm 0.03$ & $5.49 \pm 0.03$ \\
\hline UGC 4188 & $7.05 \pm 0.05$ & $6.30 \pm 0.03$ & $6.75 \pm 0.05$ & $5.78 \pm 0.03$ \\
\hline UGC 4551 & $6.64 \pm 0.02$ & $6.41 \pm 0.02$ & $6.53 \pm 0.02$ & $6.04 \pm 0.01$ \\
\hline NGC 2675 & $6.63 \pm 0.04$ & $6.37 \pm 0.03$ & $6.64 \pm 0.04$ & $5.98 \pm 0.02$ \\
\hline IC 522 & $6.85 \pm 0.04$ & $5.93 \pm 0.02$ & $6.58 \pm 0.04$ & $5.52 \pm 0.02$ \\
\hline NGC 2693 & $6.12 \pm 0.01$ & $6.05 \pm 0.01$ & $5.75 \pm 0.01$ & $5.64 \pm 0.01$ \\
\hline UGC 4702 & $6.55 \pm 0.04$ & $6.05 \pm 0.03$ & $6.27 \pm 0.04$ & $5.65 \pm 0.03$ \\
\hline NGC 2768 & $6.74 \pm 0.01$ & $6.26 \pm 0.01$ & $6.18 \pm 0.01$ & $5.87 \pm 0.0$ \\
\hline NGC 3265 & $3.59 \pm 0.01$ & $3.66 \pm 0.01$ & $3.34 \pm 0.01$ & $3.39 \pm 0.01$ \\
\hline NGC 3377 & $6.18 \pm 0.01$ & $5.57 \pm 0.01$ & $5.75 \pm 0.01$ & $5.33 \pm 0.0$ \\
\hline UGC 5928 & $6.09 \pm 0.05$ & $5.78 \pm 0.03$ & $6.09 \pm 0.05$ & $5.58 \pm 0.03$ \\
\hline NGC 3522 & $5.60 \pm 0.02$ & $5.12 \pm 0.01$ & $5.90 \pm 0.02$ & $5.17 \pm 0.01$ \\
\hline NGC 3539 & $7.36 \pm 0.09$ & $6.49 \pm 0.04$ & $7.00 \pm 0.09$ & $5.96 \pm 0.04$ \\
\hline UGC 6435 & $6.46 \pm 0.03$ & $5.96 \pm 0.02$ & $6.31 \pm 0.03$ & $5.63 \pm 0.02$ \\
\hline UGC 6683 & $6.59 \pm 0.05$ & $6.42 \pm 0.04$ & $6.61 \pm 0.05$ & $6.17 \pm 0.03$ \\
\hline NGC 3844 & $6.91 \pm 0.05$ & $6.54 \pm 0.03$ & $6.72 \pm 0.05$ & $6.20 \pm 0.03$ \\
\hline NGC 4187 & $6.81 \pm 0.03$ & $6.35 \pm 0.02$ & $6.25 \pm 0.02$ & $5.93 \pm 0.02$ \\
\hline NGC 4458 & $5.88 \pm 0.01$ & $5.47 \pm 0.01$ & $5.77 \pm 0.01$ & $5.41 \pm 0.01$ \\
\hline NGC 4478 & $6.38 \pm 0.01$ & $6.07 \pm 0.01$ & $6.17 \pm 0.01$ & $5.85 \pm 0.01$ \\
\hline M87 & $5.55 \pm 0.01$ & $5.65 \pm 0.01$ & $5.19 \pm 0.01$ & $5.35 \pm 0.01$ \\
\hline IC 3457 & $4.62 \pm 0.01$ & $4.56 \pm 0.01$ & $4.85 \pm 0.01$ & $4.71 \pm 0.01$ \\
\hline NGC 4787 & $6.56 \pm 0.05$ & $5.97 \pm 0.03$ & $6.53 \pm 0.05$ & $5.70 \pm 0.03$ \\
\hline NGC 4797 & $6.61 \pm 0.01$ & $6.25 \pm 0.01$ & $6.04 \pm 0.01$ & $5.79 \pm 0.01$ \\
\hline NGC 4827 & $6.20 \pm 0.01$ & $6.04 \pm 0.01$ & $6.05 \pm 0.01$ & $5.68 \pm 0.01$ \\
\hline NGC 4952 & $6.28 \pm 0.02$ & $6.11 \pm 0.02$ & $6.19 \pm 0.02$ & $5.75 \pm 0.01$ \\
\hline NGC 5004 & $6.37 \pm 0.02$ & $6.06 \pm 0.01$ & $6.24 \pm 0.02$ & $5.69 \pm 0.01$ \\
\hline NGC 5173 & $4.61 \pm 0.01$ & $4.31 \pm 0.01$ & $4.67 \pm 0.01$ & $4.23 \pm 0.01$ \\
\hline NGC 5329 & $6.29 \pm 0.01$ & $5.99 \pm 0.01$ & $6.01 \pm 0.01$ & $5.68 \pm 0.01$ \\
\hline UGC 8986 & $5.19 \pm 0.01$ & $4.81 \pm 0.01$ & $5.32 \pm 0.01$ & $4.96 \pm 0.01$ \\
\hline NGC 5576 & $6.05 \pm 0.01$ & $5.71 \pm 0.01$ & $5.86 \pm 0.01$ & $5.52 \pm 0.01$ \\
\hline NGC 5638 & $6.35 \pm 0.01$ & $5.96 \pm 0.01$ & $5.99 \pm 0.01$ & $5.71 \pm 0.01$ \\
\hline IC 1024 & $4.75 \pm 0.02$ & $4.32 \pm 0.01$ & $3.64 \pm 0.01$ & $3.64 \pm 0.01$ \\
\hline IC 1071 & $6.98 \pm 0.02$ & $6.51 \pm 0.02$ & $6.37 \pm 0.02$ & $5.98 \pm 0.02$ \\
\hline NGC 5813 & $6.47 \pm 0.01$ & $6.14 \pm 0.01$ & $6.00 \pm 0.01$ & $5.72 \pm 0.01$ \\
\hline UGC 10261 & $6.63 \pm 0.02$ & $6.21 \pm 0.01$ & $6.09 \pm 0.01$ & $5.65 \pm 0.01$ \\
\hline Mrk 501 & $2.56 \pm 0.01$ & $3.09 \pm 0.01$ & $2.18 \pm 0.01$ & $2.46 \pm 0.01$ \\
\hline NGC 6364 & $6.71 \pm 0.02$ & $6.27 \pm 0.01$ & $6.47 \pm 0.02$ & $5.91 \pm 0.01$ \\
\hline UGC 10935 & $6.92 \pm 0.08$ & $5.89 \pm 0.04$ & $6.39 \pm 0.08$ & $5.43 \pm 0.04$ \\
\hline NGC 7317 & $6.96 \pm 0.02$ & $6.78 \pm 0.02$ & $6.74 \pm 0.02$ & $6.18 \pm 0.02$ \\
\hline NGC 7432 & $6.63 \pm 0.03$ & $6.29 \pm 0.02$ & $6.21 \pm 0.03$ & $5.82 \pm 0.02$ \\
\hline
\end{tabular}

Notes.

${ }^{\text {a }}$ Colors for NUV $-[3.4]$ and NUV $-r$ measured at the $r$-band fractional radii $R / R_{90}=0.3$. See Figure 2 .

${ }^{\mathrm{b}}$ Colors for NUV - [3.4] and NUV $-r$ measured at the $r$-band fractional radii $R / R_{90}=0.7$. See Figure 2 .

to isolate at any temperature or age in these plots. However, the inner mean color is consistent with having little to no BHB population.

Additionally, it appears that temperature is only important at the extreme end $\Delta_{T}=0.4$. Despite the degeneracies, we find indications that age is primarily responsible for the color difference in our ETG sample. From just the three sampled ages, we show the progression of the different modeled stellar populations that might lead to color differences.

To explore the premise that age is the main cause of the color differences in the ETGs, we take isochrones of the synthetic photometry in Figure 4 at $t_{\text {age }}=2,5$, and 10 (white vertical lines in Figure 4), and plot them in FUV - NUV, NUV $-r$, NUV - [3.4] color-space. We do this in Figure 5 by plotting FSPS lines on top of the gray-scale contours that include both the $R_{\text {in }}$ and $R_{\text {out }}$ colors of the ETG sample. We outline the distribution of colors for $R_{\text {out }}$ with the dashed contour lines. We also add dust vectors (orange arrows crossing the $Z=1$ and $1.5 Z_{\odot}$ tracks) with an increasing dust parameter from $0-1$ using the Charlot \& Fall (2000) dust models. The top, middle, and bottom rows are the isochrones at ages 2, 5, and $10 \mathrm{Gyr}$ as labeled.

Our first assessment is that the density peaks are clearly separated, and dust may be ruled out simply by observing the direction of the vectors in Figure 5. If dust was preferentially reddening the inner half-light of the ETGs, the FUV - NUV $R_{\text {in }}$ colors would lie redward. We do not mean that these are entirely dustless (we actually include dust in the FSPS models; see Section 3). Rather, we assume that dust is not a strong enough factor to cause the color difference.

The 2 Gyr plots (top row in Figure 5) indicate that the colors for the inner regions could only be explained by super-solar metallicities $>1.5 Z_{\odot}$. At the same age, the outer regions are more likely to host a $1-1.5 Z_{\odot}$ stellar population with a moderate BHB fraction. At $5 \mathrm{Gyr}$ (middle plots), the $R_{\text {in }}$ colors could be caused by higher metallicity/moderate BHB fractions and the outer regions would have a lower metallicity and higher numbers of BHBs. At $10 \mathrm{Gyr}$, the isochrones give narrower ranges and the metallicity dependence is stark: $R_{\text {in }}$ would be dominated by a $1-1.5 Z_{\odot}$ stellar population and an insignificant BHB fraction; $R_{\text {out }}$ would be dominated by a $<1 Z_{\odot}$ stellar population and may include slightly more BHBs. We also note that for 2-5 Gyr the dominating SFH is $\tau=0.2-0.6$; at $10 \mathrm{Gyr}$ the range is widened to all $\tau$.

The above conditions indicate two possible scenarios: (1) if the bulk of the bulge and disk mass coevolved, the metallicities must be different by $\sim 1 Z_{\odot}$ and (2) the ETGs formed in an inside-out process with at least two major stages of growth $\gtrsim 1$ Gyr apart. These appear to be two distinctly different mechanisms of formation, and we deepen our analysis in the following section to determine the likelihood of either scenario.

\subsection{Age Estimates for $R_{\text {in }}$ and $R_{\text {out }}$}

To constrain the likelihood of the results based on Figure 5, we estimate the ages for the inner and outer regions of the ETGs. However, we showed in the previous section that the high degeneracy of the synthetic photometry makes it difficult to derive accurate ages for each of the objects (e.g., Figure 4). In order to check the results, given the degeneracies, we resample the data to find the most likely statistical fit to the ages given a fixed set of parameters. We fit the data to the FSPS templates, with the FUV - NUV, NUV $-r$, and NUV - [3.4] colors. We resampled the inner and outer colors by replacement estimates (bootstrapping) within the errors for 500 iterations to estimate errors and probabilities for the $\chi^{2}$ fitting. The $\chi^{2}$ fitting was done between the resampled observed colors and the FSPS data. After minimizing the $\chi^{2}$ for each iteration, we used the maximum likelihood for each parameter combination. The probability distribution functions were used to estimate the 


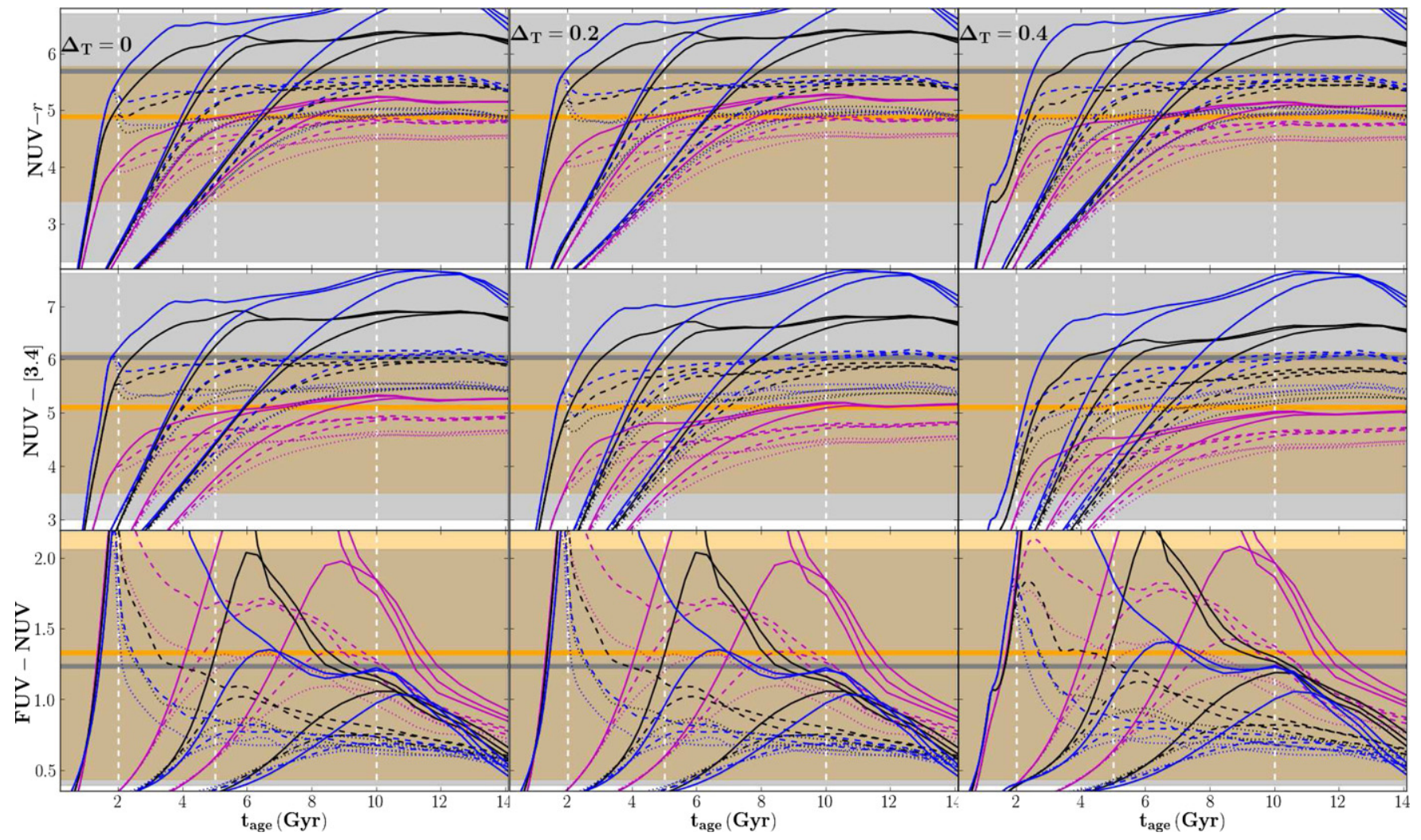

Figure 4. To illustrate the significant degeneracies with age, we plot color vs. age from $0.2<t_{\text {age }}<14.1$ Gyr, with the ETG sample (orange and gray shading), and the FSPS colors (NUV $-r$, NUV - [3.4], FUV - NUV) with temperature boosts $\Delta_{T}=0,0.2$, and 0.4 from left to right (as labeled). The magenta, black, and blue lines are $Z=0.25,1.0$, and $1.5 Z_{\odot}$, respectively. The solid, dashed, and dotted lines represent the $0,0.2$, and $0.5 \mathrm{BHB}$ fractions initiated at $t_{\mathrm{age}}=2 \mathrm{Gyr}$. Each line style has three lines (not labeled) for the three star formation histories $(\tau=0.2,0.6,1.0)$. The range of color for the inner and outer regions of the ETGs are shaded in gray and orange bands, respectively. The solid horizontal lines indicate the mean values for the respective regions. The white vertical lines are placed at 2,5 , and 10 Gyr, which we inspect in Figure 5.

Table 3

Possible Parameter Solutions for the Mean $R_{\text {in }}$ and $R_{\text {out }}$ Colors at 2, 5, and $10 \mathrm{Gyr}$

\begin{tabular}{|c|c|c|c|c|c|c|c|c|c|}
\hline \multirow[t]{2}{*}{$t_{\mathrm{age}}$} & \multicolumn{3}{|c|}{$\Delta_{T}=0$} & \multicolumn{3}{|c|}{$\Delta_{T}=0.2$} & \multicolumn{3}{|c|}{$\Delta_{T}=0.4$} \\
\hline & $Z\left(Z_{\odot}\right)$ & $\tau(\mathrm{Gyr})$ & $f_{\mathrm{BHB}}$ & $Z\left(Z_{\odot}\right)$ & $\tau(\mathrm{Gyr})$ & $f_{\mathrm{BHB}}$ & $Z\left(Z_{\odot}\right)$ & $\tau(\mathrm{Gyr})$ & $f_{\mathrm{BHB}}$ \\
\hline \multicolumn{10}{|c|}{$R_{\text {in }}$} \\
\hline $2 \mathrm{Gyr}$ & 1.5 & 0.2 & $<0.25$ & 1.5 & 0.2 & $<0.25$ & $\ldots$ & $\ldots$ & $\ldots$ \\
\hline $5 \mathrm{Gyr}$ & $1-1.5$ & 0.6 & $0-0.5$ & $1-1.5$ & $0.2-0.6$ & $<0.25$ & $1-1.5$ & $0.2-0.6$ & $<0.25$ \\
\hline $10 \mathrm{Gyr}$ & $0.25-1.5$ & $0.2-1$ & $0-0.5$ & $1-1.5$ & $0.2-1$ & $<0.25$ & $1-1.5$ & $0.2-1$ & $<0.25$ \\
\hline \multicolumn{10}{|c|}{$R_{\text {out }}$} \\
\hline $2 \mathrm{Gyr}$ & $1-1.5$ & 0.2 & $0-0.5$ & $1-1.5$ & 0.2 & $>0.25$ & $1-1.5$ & 0.2 & $0-0.5$ \\
\hline $5 \mathrm{Gyr}$ & $0.25-1.5$ & $0.2-0.6$ & $0-0.5$ & $0.25-1$ & $0.2-0.6$ & $0-0.5$ & $0.25-1$ & $0.2-0.6$ & $0-0.5$ \\
\hline $10 \mathrm{Gyr}$ & $<1$ & $0.2-1$ & $0-0.5$ & $0.25-1$ & $0.2-1$ & $0-0.5$ & $0.25-1$ & $0.2-1$ & $0-0.5$ \\
\hline
\end{tabular}

weighted average age. The ages and errors were calculated for each BHB, $\tau, Z$, and $\Delta_{T}$ combination for each object.

In Figure 6 we show the effects that BHB fraction, metallicity, and SFH have on the estimated ages $\left(t_{\text {age }}(\right.$ in $)$ and $t_{\text {age }}($ out $\left.)\right)$. Each data point is a weighted average of the entire ETG sample, testing different parameter configurations (e.g., one point for $f_{\mathrm{BHB}}=0, \tau=0.2$, and $Z=1 Z_{\odot}$ ). We do not separate the temperature boosts here since Figure 4 indicates that the effects on color are negligible for this analysis.

The points reflect the assumption that both the inner and outer regions are evolving with the same set of parameters. We are essentially testing one condition for the first scenario in Section 4.2. For example, the magenta square $\left(Z=1.5 Z_{\odot}\right.$, $f_{\mathrm{BHB}}=0.5$, and $\tau=1$ ), easily identified as the outlier, is fixing the prior that the parameter combinations are the same for the inner and outer regions. In other words, each point is the $t_{\text {age }}$ coordinate for the inner and outer regions having the same metallicity, BHB fraction, and $\tau$. With this assumption, we find that nearly all points are below the equality line, and many (12 points) have $\Delta t_{\text {age }}>1$ Gyr.

In order for the inner and outer regions to have equal ages, they must have a certain set of properties. Over half of the points within errors of the equal age line have low metallicity (double circles for $Z=0.25 Z_{\odot}$ ) and some fraction of BHBs (diamonds and squares). This constrains our hypothetical scenarios mentioned in the previous section.

Average ages derived from low metallicity $\left(0.25 Z_{\odot}\right)$ show the least difference between $t_{\text {age }}$ (in) and $t_{\text {age }}$ (out). The average 


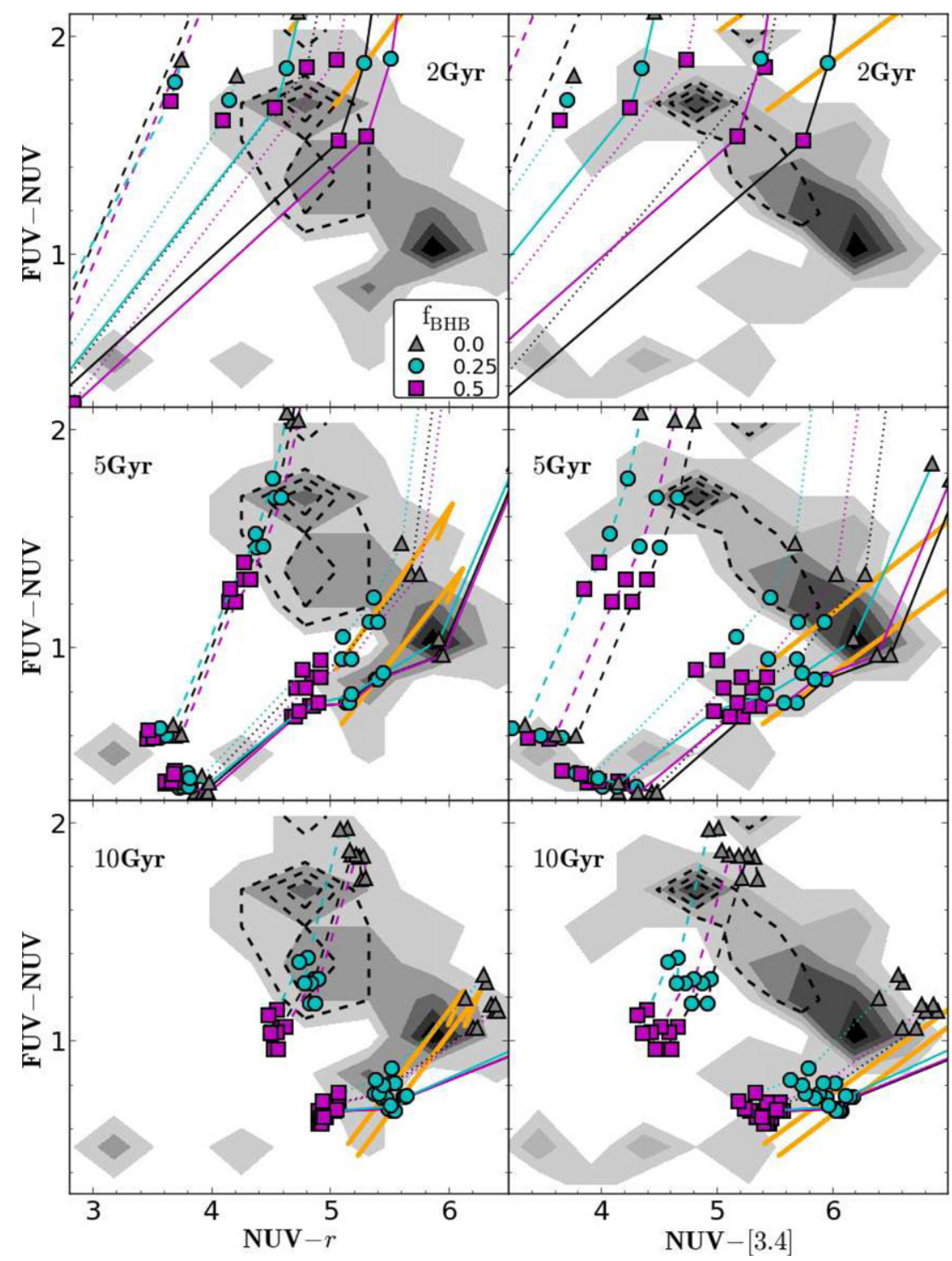

Figure 5. FUV - NUV, NUV - $r$, and NUV - [3.4] plots with the distribution of inner/outer colors, and isochrones at 2 (top), 5 (middle), and 10 (bottom) Gyr. The gray-filled contours include the inner and outer radii colors, while the outer regions are delineated with dashed contour lines. The peaks of the two regions are clearly separated in color-space. The dashed, dotted, and solid FSPS lines are $Z=0.25,1$, and $1.5 Z_{\odot}$, respectively. The temperature increases are $\Delta_{T}=0$ (black), 0.2 (magenta), 0.4 (cyan). All lines include SFH $e$-folding times $\tau=0.2,0.6$, and $1 \mathrm{Gyr}$. The BHB fraction at points along the isochrone tracks are marked by the symbols as labeled. We include dust vectors (orange) with an increasing dust parameter 0-1, using a Charlot \& Fall (2000) dust model.

age separation is $0.28,1.13$, and 0.9 Gyr for $Z=0.25,1$, and $1.5 Z_{\odot}$, respectively. When selected by BHB fraction, the average differences are 1.21, 0.86, and $0.24 \mathrm{Gyr}$ for $f_{\mathrm{BHB}}=0$, 0.25 , and 0.5 , respectively; $\tau$ shows little difference $0.83,0.8$, and $0.69 \mathrm{Gyr}$ for $\tau=0.2,0.6$ and 1, respectively.

Given these priors, $67 \%$ of the ages for the inner regions are older (to the right of the shaded region), and have an average age difference of $1.1 \mathrm{Gyr}$. The mean difference for all points is $0.8 \mathrm{Gyr}$ with a range of -0.3 to $1.9 \mathrm{Gyr}$ if the parameters are assumed to be equal. The average age for the inner region is $7.0 \pm 0.3 \mathrm{Gyr}$ and the outer is $6.2 \pm 0.2 \mathrm{Gyr}$. The assumptions we used to derive these results indicate that the outer regions are likely to have formed $0.5-1.2 \mathrm{Gyr}$ after the inner regions.

\subsection{Age-Metallicity Ratios}

We explore the possibility of an age-metallicity difference between $R_{\text {in }}$ and $R_{\text {out }}$ in our sample. Since we are limited to three metallicities, the results we present are a crude estimate of how metallicity and age behave between these regions. Figure 7 shows the inner to outer average age ratios for different $Z_{\text {inner }}$ and $Z_{\text {outer }}$ metallicity combinations. The error bars span the range of ages for the set of parameters at that metallicity ratio (e.g., $\mathrm{BHB}, \tau)$. We fit the $\Delta \log (\mathrm{Age})-\Delta \log (Z)$ values with the following result:

$$
\Delta \log (\text { Age })=-0.16 \Delta \log (Z) .
$$

Figure 7 shows that, for $Z_{\text {inner }} / Z_{\text {outer }}<1$, it is more likely that the stellar populations in the inner half-lights of our sample are older than the outer even though the range of ages spans widely about the mean. For $Z_{\text {inner }} / Z_{\text {outer }}>1$, the ages span a narrow range around $t_{\text {inner }} / t_{\text {outer }}=1$. For metallicity gradients of decreasing $Z$ with radius, the estimated age difference for the ETGs increases, resulting in an average of $1.4 \mathrm{Gyr}$ (older toward the centers), which is nearly double the average when we assumed $Z_{\text {inner }} / Z_{\text {outer }}=1$ (see Section 4.3). This further supports the hypothesis that the color differences are likely due 


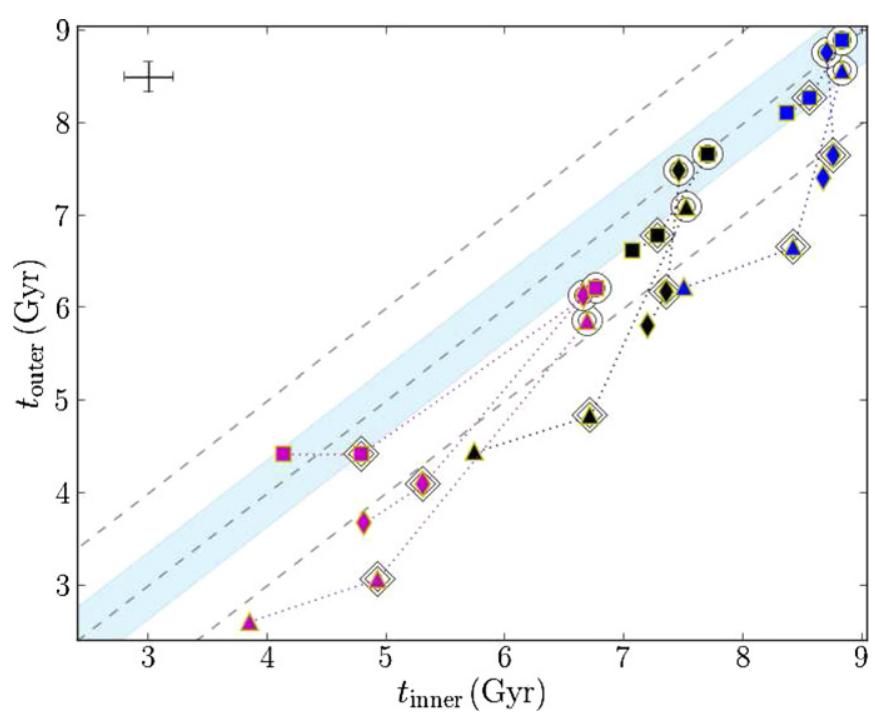

Figure 6. Estimated ages in Gyr for $R_{\text {in }}$ and $R_{\text {out }}$ considering the different CSP parameter combinations, assuming homogeneity of the stellar populations in the inner and outer regions. The ages are combined weighted averages for the inner and outer regions for the 49 ETGs in our sample (one symbol is the average for all ETGs). The different triangles, diamonds, and squares represent $f_{\mathrm{BHB}}=0$, 0.25 , and 0.5 , respectively. The black, blue, and magenta colors are $\tau=0.2$, 0.6 , and 1, respectively. Symbols on double circles and double diamonds have $Z=0.25$ and $1 Z_{\odot}$, respectively, while all other symbols have $Z=1.5 Z_{\odot}$. The dotted lines trace metallicities along a fixed $f_{\mathrm{BHB}}$ and $\tau$ with metallicity. The shaded region highlights the equal age line within the average error. The average error is illustrated by the error bars in the upper left corner. We include equal age \pm 1 Gyr dashed lines for gauging the age differences.

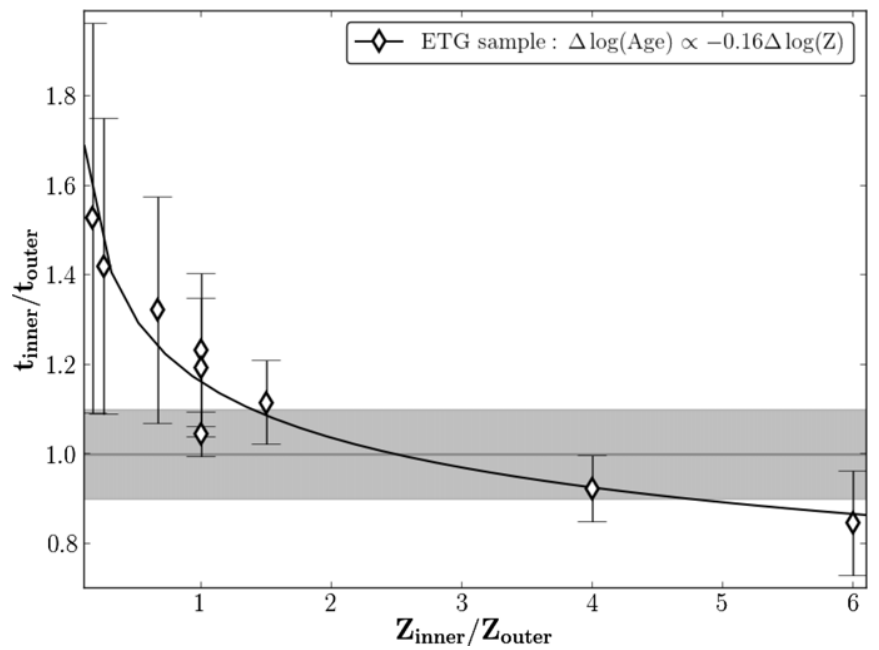

Figure 7. We plot the estimated age ratios for each metallicity combination between the inner and outer regions of the ETGs. The solid line is the fit of the ETG sample (diamonds). The error bars show the range of ages for the different $\mathrm{BHB}, \tau$ combinations. The horizontal gray area shades the equal age line within a $10 \%$ margin.

to multi-stage evolution and less of a metallicity difference. However, the information presented here is not conclusive of a presence, or lack thereof, of a metallicity gradient.

\subsection{BHBs and the Color NUV - [3.4] Color Difference}

The majority of our analysis is dedicated to determining whether the color difference is indicative of co-evolving or multi-stage evolution. We also want to know the source of UV emission in these ETGs since we have shown our sample is unlikely to have undergone recent star formation. The age estimates indicate that significant UV emission is largely produced

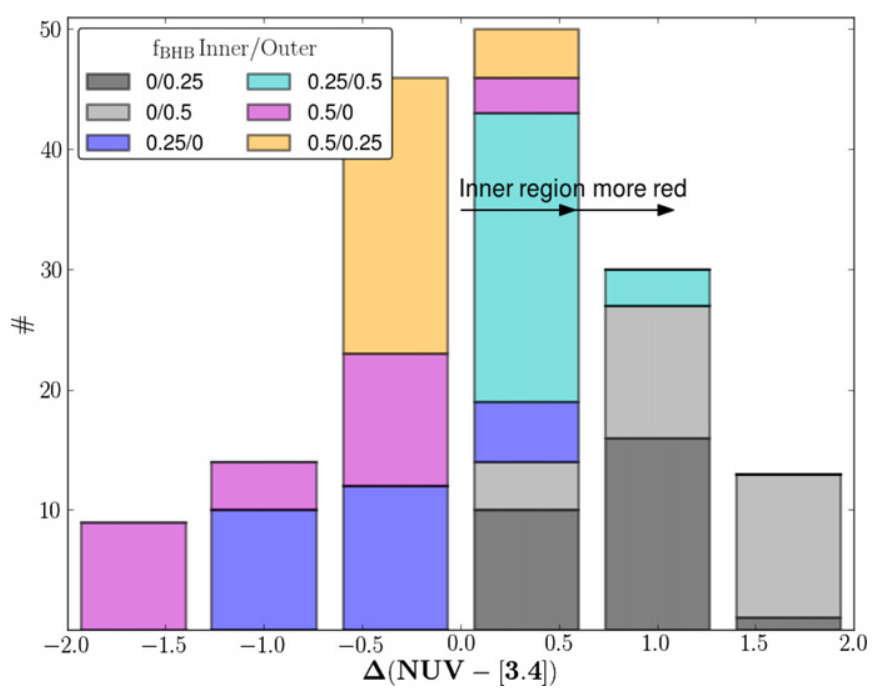

Figure 8. We show the stacked distributions of $\Delta$ (NUV - [3.4]) determined by imposing different BHB fractions at the inner and outer radii as labeled. For example, the dark gray labeled $0 / 0.25$ refers to the color at $f_{\mathrm{BHB}}=0$ and 0.25 for $R_{\text {in }}$ and $R_{\text {out }}$, respectively. The models with higher BHB fraction in the outer radii are more likely to have a $1 \mathrm{mag}$ color difference (light blue, dark gray, and light gray).

by sources in an older stellar population $\left(t_{\text {age }}>2\right.$ Gyr; see Figures 5 and 6). One question we asked is whether a significant fraction of BHB stars can accomplish this. Can the overall trend of bluer NUV - [3.4] and NUV $-r$ with increasing galactocentric radius be due to the presence of $\gtrsim 25 \%$ BHBs in a 2-5 Gyr old stellar population? For example, in Figure 5, at 10 Gyr the BHB fraction is important only within a small area of color-space: $0.6<\mathrm{FUV}-\mathrm{NUV}<1.5$, $4.4<\mathrm{NUV}-r<5.7,4.2<\mathrm{NUV}-[3.4]<6.3$. The mean $R_{\text {in }} / R_{\text {out }}$ values are: $(\mathrm{FUV}-\mathrm{NUV})=1.2 / 1.3,(\mathrm{NUV}-r)=$ $5.7 / 4.9$, and (NUV $-[3.4])=6.1 / 5.1$, which do fall into these ranges of color-space, indicating that $\mathrm{BHBs}$ could be a source of the UV emission.

To investigate how they may contribute to the 1 mag color difference between $R_{\text {in }}$ and $R_{\text {out }}$, we assign different $\mathrm{BHB}$ fractions between the inner and outer radii and extract the corresponding NUV and [3.4] photometry from FSPS templates using the estimated ages for the ETG sample at $R_{\text {in }}$ and $R_{\text {out }}$ (see Section 4.4). Figure 8 shows the stacked distributions of $\left.\Delta(\mathrm{NUV}-[3.4])=(\mathrm{NUV}-[3.4])_{\text {inner }}-(\mathrm{NUV}-[3.4])_{\text {outer }}\right)$. Higher $f_{\mathrm{BHB}}$ in the outer regions are most likely to make colors redder in the centers. In this case, a jump of $f_{\mathrm{BHB}}=0.25$ in the outer region produces this effect.

\subsection{Mass, Star Formation, and UV-Mid-IR Color}

We explore whether the colors in the inner and outer radii are related to star formation rates and stellar mass (SFR and $M^{*}$, respectively) derived from integrated photometry (e.g., for lowsurface-brightness or high-redshift sources). We first compute SFRs and stellar masses from the integrated magnitudes (for the whole system in each case) using the UV calibration of Kennicutt (1998, Equation (1)) and the 2MASS $K_{\mathrm{s}}$ versus $g-r$ calibration of Bell et al. (2003).

In Figure 9 we plot these values against the NUV - [3.4] colors for the individual sources and find that (1) the SFRs show a scatter for both the inner and outer colors and (2) there is an increase in stellar mass with redder color. This increase in mass with more red ETGs is consistent with other observations 

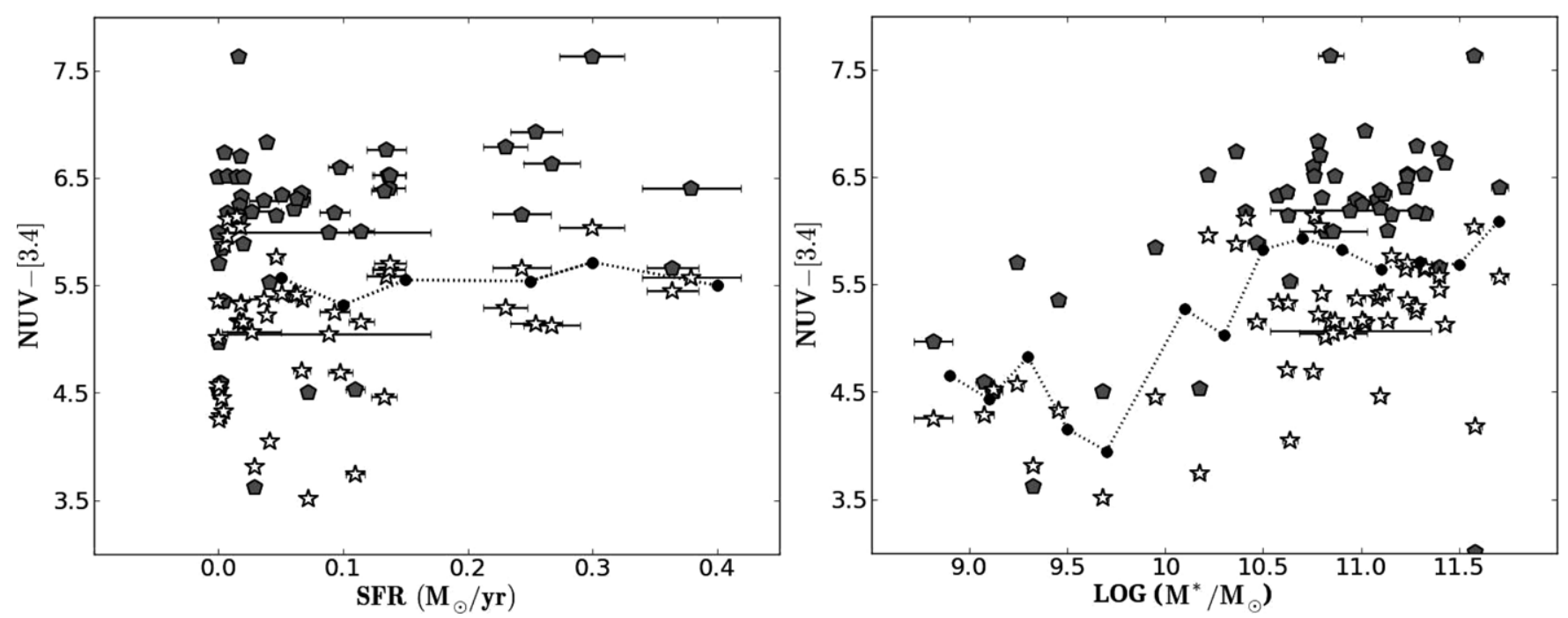

Figure 9. NUV - [3.4] inner (gray pentagons) and outer (stars) region colors as a function of star formation rate (left) and stellar mass (right). The black circles and dotted line show the binned averages of the total flux NUV - [3.4] colors for SFR and $M^{*}\left(\Delta \mathrm{SFR}=0.05 M_{\odot} \mathrm{yr}^{-1}\right.$ and $\left.\Delta \log \left(M^{*} / M_{\odot}\right)=0.2\right)$. As expected, the ETGs are quiescent, and there appears to be no correlation between the UV-mid-IR color and SFR. The average error for NUV - [3.4] is $0.07 \mathrm{mag}$. The symbols are larger than the photometric errors.

(e.g., Bell et al. 2003). The binned averages are the total flux colors (not inner and outer but the entire galaxy), averaged over SFR and stellar mass bins. The SFR is very low for all of the galaxies; therefore, we can assume the bluer colors for the outer radii have not been created by recent star formation. The majority of the ETGs are within the massive galaxy range $\left(>10^{11} M_{\odot}\right)$. We note that the Bell et al. (2003) analysis does not include contributions of HB stars to the UV. However, it is useful to discern if the color difference could be caused by a primarily young stellar population.

\section{DISCUSSION}

We have shown that a clear difference in the UV to IR color between the inner and outer regions of our sample of $49 \mathrm{E} / \mathrm{S} 0$ galaxies exists. Moreover, we see no significant variation between the E and S0 Hubble types. In this section, we discuss which stellar mass assembly histories are consistent with these observations based on our analysis in the previous section. The CSP predictions of color based on BHBs, ages, and metallicities illustrate that secular coevolution of a bulge and disk progenitor would be difficult to explain without a significant internal mixing mechanism.

The first result we found was the 1 mag difference between the colors measured for the inner half-light and the outer radii. We hypothesized that a different stellar population must be dominating the respective regions since color gradients in ETGs and globular clusters have been repeatedly measured. For example, Carter et al. (2011) use GALEX and 2MASS to discuss the radial UVX in ETGs; they find a steep gradient between the inner and outer regions with an increase in UVX at the core. They attribute this to increased $\alpha$ enhancement from Type II $\mathrm{SNe}$, and perhaps $\mathrm{He}$ abundance, rather than to dry mergers in an outside-in scenario. Moreover, recent papers by Forbes et al. (2011) and Arnold et al. (2011) present optical color radial profiles for a nearby globular cluster (NGC 1407 and S0 galaxy NGC 3115, respectively). Both show evidence of a two-stage formation history using color analysis with $g-i$. They find a color difference and decrease in metallicity $[\mathrm{Fe} / \mathrm{H}]$ between the inner and outer regions.
We took advantage of the better-defined separations of the FSPS tracks in the NUV - [3.4] color-space, and augmented this with the NUV $-r$ and FUV - NUV color to narrow the possibilities between the well-known degeneracies. In the density plots (Figure 5), the separate peaks for inner and outer fall in regions depending on the assumed age, and support the idea that EHBs are the possible source of UV emission. Furthermore, this is consistent with being driven primarily by differences in age with secondary contributions from differences in BHB fraction, metallicity, and SFH.

Based on these assessments, and as we mentioned in Section 4.2, two possible formation scenarios are consistent with comparison of the FSPS colors and the different colors of the inner and outer regions in the ETG sample: (a) if the bulge and disk coevolved, the metallicities must be different by $\gtrsim 1 Z_{\odot}$ and (b) the ETGs formed in an inside-out process with at least two major stages of growth $\gtrsim 1$ Gyr apart.

For the first case, the inner parts of the galaxy would need to exhibit a strong metallicity gradient within a short time-frame through self-enrichment or merging. If the hierarchical model accurately describes the formation of the bulk of the bulge mass, then the timescales for relaxation are on the order of 0.1-1 Gyr (e.g., Barnes \& Hernquist 1996), and pseudobulge formation at timescales $>2$ Gyr. Surveys of globular clusters in M31 show a mix of metal-poor to metal-rich clusters across the galaxy with metal-rich clusters predominately in the central $10 \mathrm{kpc}$ radius (Saito \& Iye 2000; Perrett et al. 2002). Saito \& Iye (2000) model the timescale to produce a suitable metallicity gradient over the galactocentric radius, and calculate that self-enrichment with a collapsing disk would take $\sim 5 \mathrm{Gyr}$, while a merging event would take $\sim 2$ Gyr. Both surveys conclude that merging with low-metallicity galaxies is most likely to have produced the metallicity gradients, supporting multi-stage evolution. If this is typical for most ETGs, evidence of a second-stage or multi-stage event would be expected. The timescales of self-enrichment are too long for the inner and outer stellar populations to have coevolved, which leads us to the second scenario.

Our results are more consistent with an inside-out multistage evolution. Whether or not the color difference between the inner and outer radii is caused by a metallicity gradient, the 
centers of the ETGs must be on average 1 Gyr older, according to our results. The question remains what merger process, gas-rich or gas-poor, is most likely to produce the age/color differences observed.

In principle, several processes can contribute to stellar mass assembly in local massive galaxies. First, in situ star formation may be triggered by accretion of gas from the intergalactic medium (IGM). Mergers may also play a part through "wet" (dissipational) mergers between gas-rich galaxies, or "dry" (dissipationless) mergers between gas-poor galaxies. The stellar ages for wet mergers tend to be younger, supplying the gas for starbursts. A "frosting" of gas-rich mergers is often used to explain the dispersion in ages within ETGs (Trager et al. 2000; Sánchez-Blázquez et al. 2006, 2009). For dry mergers, the most massive bulge-dominated ETGs form by the accretion of galaxies with older stellar populations onto the central bulge.

Each of these may occur via major or minor mergers, and observational evidence varies on which is most influential in terms of mass building (e.g., van Dokkum 2005; Cox et al. 2006; Sánchez-Blázquez et al. 2009). The analysis in van Dokkum (2005) shows that $71 \%$ of bulge-dominated galaxies have some signature of interaction (i.e., tidal disturbances), and 35\% have had a recent dry major merger. Sánchez-Blázquez et al. (2009) did a spectral analysis of the van Dokkum (2005) sample and find that the sample easily divides into a range of wet to dry merger stages in age and metallicity. The metallicities are $1-1.5 Z_{\odot}$ for all merger scenarios (Sánchez-Blázquez et al. 2006, 2009), but the dry mergers tend toward slightly higher metallicities and ages $\gtrsim 5$ Gyr. Simulations by Cox et al. (2006) show that wet mergers leave remnants that are smaller, with higher rotation, and a more disk-like shape. Boxy, massive ellipticals can result from dry mergers, but the observed nearby ETGs are likely formed by a combination of wet/dry merging. Our sample consists of more massive ellipticals, and the estimated ages $(\sim 7 \mathrm{Gyr})$ support dry merging for $z \lesssim 0.3$. If a metallicity gradient exists, dry merging with higher metallicity galaxies may be consistent with our age- $Z$ trends. We find inconsistent trends with our first scenario, where we proposed that higher metallicities at the center would cause the red colors. However, our calculations will need to be verified with a more rigorous age $-Z$ radial profile fitting.

There is evidence that the contribution from wet and dry mergers may result in a distinct two-stage formation history for ETGs. Observations show that the inner and outer regions of nearby galaxies decouple at some point, implying that a wet process is producing transitions and velocity dispersions indicative of recent star formation (Treu et al. 2005; Emsellem et al. 2007; Kormendy et al. 2009; Arnold et al. 2011; Forbes et al. 2011). In an inside-out evolution, the oldest stellar populations exist in the center, and the outer halo or disk has undergone more recent star formation via minor gas-rich mergers.

Recent observations from Nelson et al. (2012) support insideout evolution for rapidly forming disks at $z \sim 1$, and agree with our age and multi-stage formation time-frames. Their results show an extended $\mathrm{H} \alpha$ disk, outside of an older central bulge, compared with the $R$-band stellar continuum $\left(r_{e}(\mathrm{H} \alpha)>\right.$ $\left.1.3 r_{e}(\mathrm{R})\right)$. Damjanov et al. (2009) compare the restframe optical radii of compact passive galaxies at $1<z<2$ with nearby ETGs. They show that the inner cores of the nearby ETGs are consistent with the sizes of the higher redshift compact galaxies.

Our results are compatible with a two- or multi-stage, insideout formation history, in which the core formed through rapid star formation via wet mergers or cold gas accretion from the
IGM at least $\sim 7$ Gyr ago $(z \gtrsim 2)$. After $\sim 1-2$ Gyr (at $z \sim 1$ ), a second phase of minor-merging would induce a new major starburst phase possibly in an outer disk of the galaxy. Our age estimates require that the inner and outer regions are both greater than 2 Gyr old, and differ by an average of 1.4 Gyr.

Additionally, the outer regions are consistent with having an elevated fraction of EHBs that can begin as early as 2-5 Gyr, based on the synthetic photometry (see Figure 4). Our results show that an increase of $0.25 \mathrm{BHB}$ fraction in the outer regions can produce the $1 \mathrm{mag}$ color difference we observe. The $\mathrm{BHB} / \mathrm{EHB}$ phases are predicted to have lifetimes of $\sim 10 \mathrm{Myr}$, so a population of stars in this phase would be very shortlived compared to the average age of $\gtrsim 4$ Gyr in the inner regions.

A possible alternative explanation is differential extinction between inner and outer regions. However, the extinction vectors (orange arrows) shown in Figure 5 imply they would need to be over a magnitude of extinction, and the FUV - NUV would show the opposite separation between the inner and outer regions, which we do not observe. It is possible but unlikely, though we cannot completely rule it out from our data. This may explain why we see a slight bias for the highly elliptical galaxies $(b / a<0.6)$ at the inner radii (see Figure 3$)$.

\section{CONCLUSIONS}

The longstanding question of what causes UVX is likely not answered by one simple population (old or young) or parameter tweak. We explore the origin of the UV emission in our sample using stellar population synthesis models. We also chose within a range of values, the parameters (BHB fraction, $Z, \tau, \Delta_{T}$ ) that best fit the UV, optical, and mid-IR colors of our sample. We then looked at the spatial breakdown of UV to optical and UV to mid-IR colors. Through this approach, the following results were observed.

1. WISE and GALEX colors FUV - NUV and NUV - [3.4] are highly effective at separating the parameters that drive the observed colors. Our CSP tracks show that, at $10 \mathrm{Gyr}$, the BHB fraction is important only within a small area of colorspace: $0.6<\mathrm{FUV}-\mathrm{NUV}<1.5,4.4<\mathrm{NUV}-r<5.7$, $4.2<\mathrm{NUV}-[3.4]<6.3$.

2. The 49 ETGs in this sample exhibit a strong color difference with bluer colors on the outside of the galaxies in both UV-optical and UV-mid-IR. We extract the photometry for the inner half-light and outer 50\%-90\% and find a clear color difference independent of E/S0 type. The average value of the ETG $R_{\text {in }} / R_{\text {out }}$ colors are NUV $-r=5.7 / 4.9$, and NUV $-[3.4]=6.1 / 5.1$.

3. Increasing the fraction of BHBs in the outer regions by 0.25 can create the observed color difference, where the inner are redder than the outer regions by 1 mag in NUV - [3.4].

4. We find that the properties of the inner and outer regions are significantly different, either in age, metallicity, and/or the existence of BHBs. We discuss two formation scenarios based on our results: (1) if the bulge and disk coevolved, the metallicities must be significantly different $\left(0.25 Z_{\odot}\right.$ in the outer regions, $>1 Z_{\odot}$ in the centers) and (2) the ETGs formed in an inside-out process with at least two major stages of growth $\gtrsim 1$ Gyr apart. Our age estimates indicate that the second scenario is most likely.

5. The average ages are estimated to be $7.0 \pm 0.3 \mathrm{Gyr}$ (inner) and $6.2 \pm 0.2 \mathrm{Gyr}$ (outer), with a minimum of $2.6 \mathrm{Gyr}$. Even when we assume homogeneity of parameters over radius, 

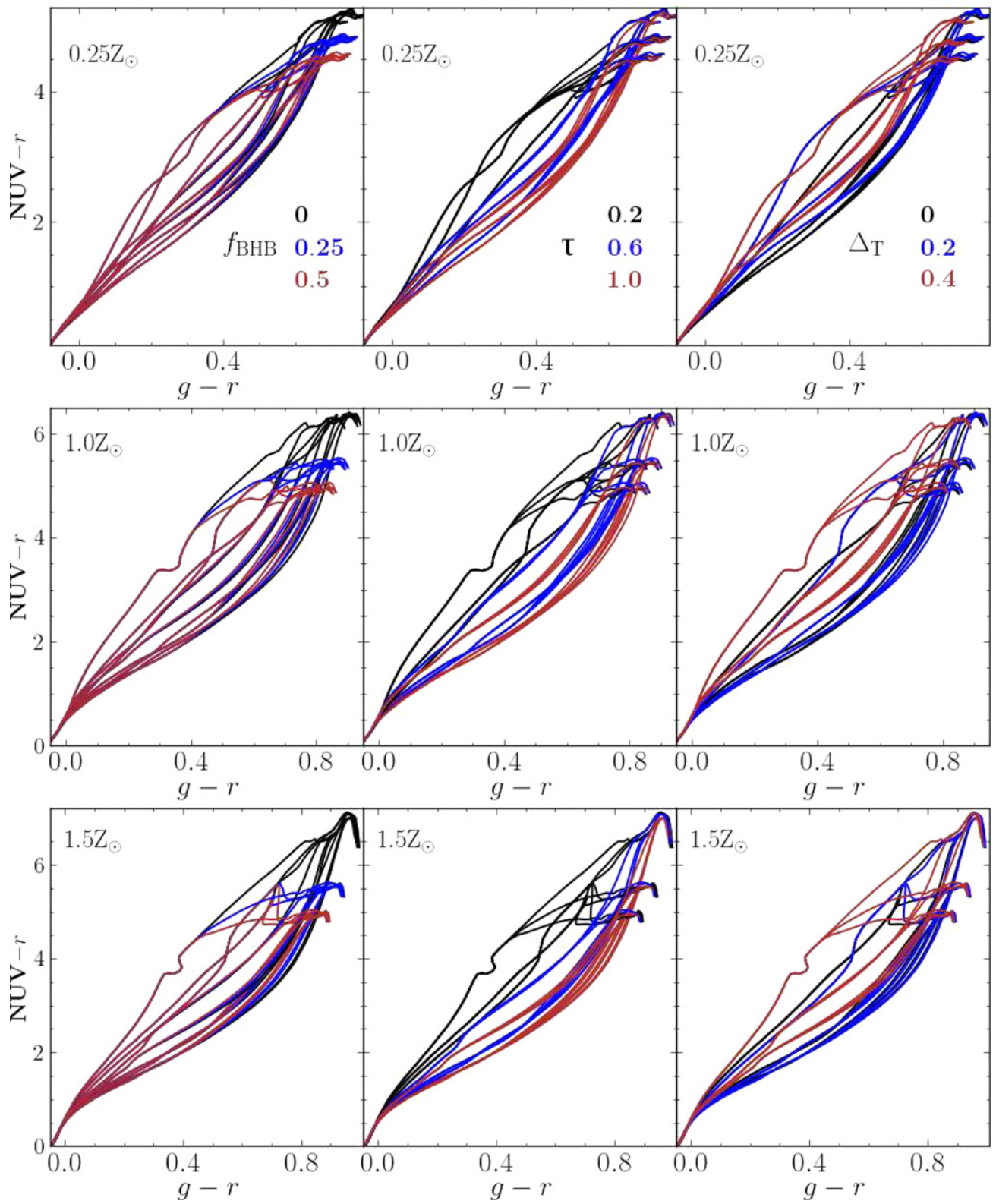

Figure 10. Flexible Stellar Population Synthesis (FSPS; Conroy et al. 2009), composite stellar population (CSP) templates depicting the evolution of NUV $-r$ vs. $g-$ $r$ color-space within an age range of $0.03-14.1$ Gyr. The three rows depict tracks for different metallicities $Z=0.25,1.0$, and $1.5 Z_{\odot}$ as labeled. Each row/metallicity shows the same templates/color-color values. The lines are colored differently (left to right) to emphasize how the parameters are evolving as labeled in the top row panels: BHB fraction, $e$-folding time $\tau$ in Gyr, and the increase in effective temperature $\Delta_{T}\left(\log \left(T_{\text {eff }}\right)\right)$ at $\mathrm{HB}$ onset.

there is evidence of multi-stage evolution where the outer regions are likely to have formed at least $\sim 0.8 \mathrm{Gyr}$ after the inner regions $\left(-0.3<\left\langle\Delta t_{\text {age }}\right\rangle<1.9 \mathrm{Gyr}\right)$.

6. The age differences estimated for metallicity gradients with an increase in $Z$ at larger radial distances are younger at the center by an average of $\sim 300 \mathrm{Myr}$. For increasing $Z$ with radius they present age differences $\left(t_{\text {age }}(\right.$ in $)-t_{\text {age }}($ out $\left.)\right)$ of 1.4 Gyr with a range of $0.6-2.2$ Gyr. This suggests that age and other stellar population properties are contributing to the color difference rather than metallicity.

7. Since the estimated ages are beyond the lifetimes of starforming regions to contribute significantly to the UV emission, we assume that BHBs or EHBs are the primary source of the UVX, agreeing with previous results (e.g., Greggio \& Renzini 1990). The average colors fall within the ranges predicted for BHB fractions greater than 0.25 in NUV $-[3.4]$ in the outer regions.
These combined results lead us to the conclusion that the UV observed in these massive ETGs is likely caused by an extreme HB phase of older stars and that the ETGs went through a multi-stage evolution that is coincident with an inside-out cessation. We do not discount that star formation is causing UV in other ETGs, but our particular selection may have biased us toward more massive ETGs that are slow rotators and not star-forming. A larger sample will be explored in detail in a subsequent paper to look at the individual properties of the galaxies and their environments in more detail. Previous studies do not probe the range of ETG type with the radially binned extended photometry, and these very extended objects would not have the coverage out to large radii that WISE naturally provides.

We thank the anonymous referee for thorough comments that greatly improved this paper. We thank Marcio Catelan for 

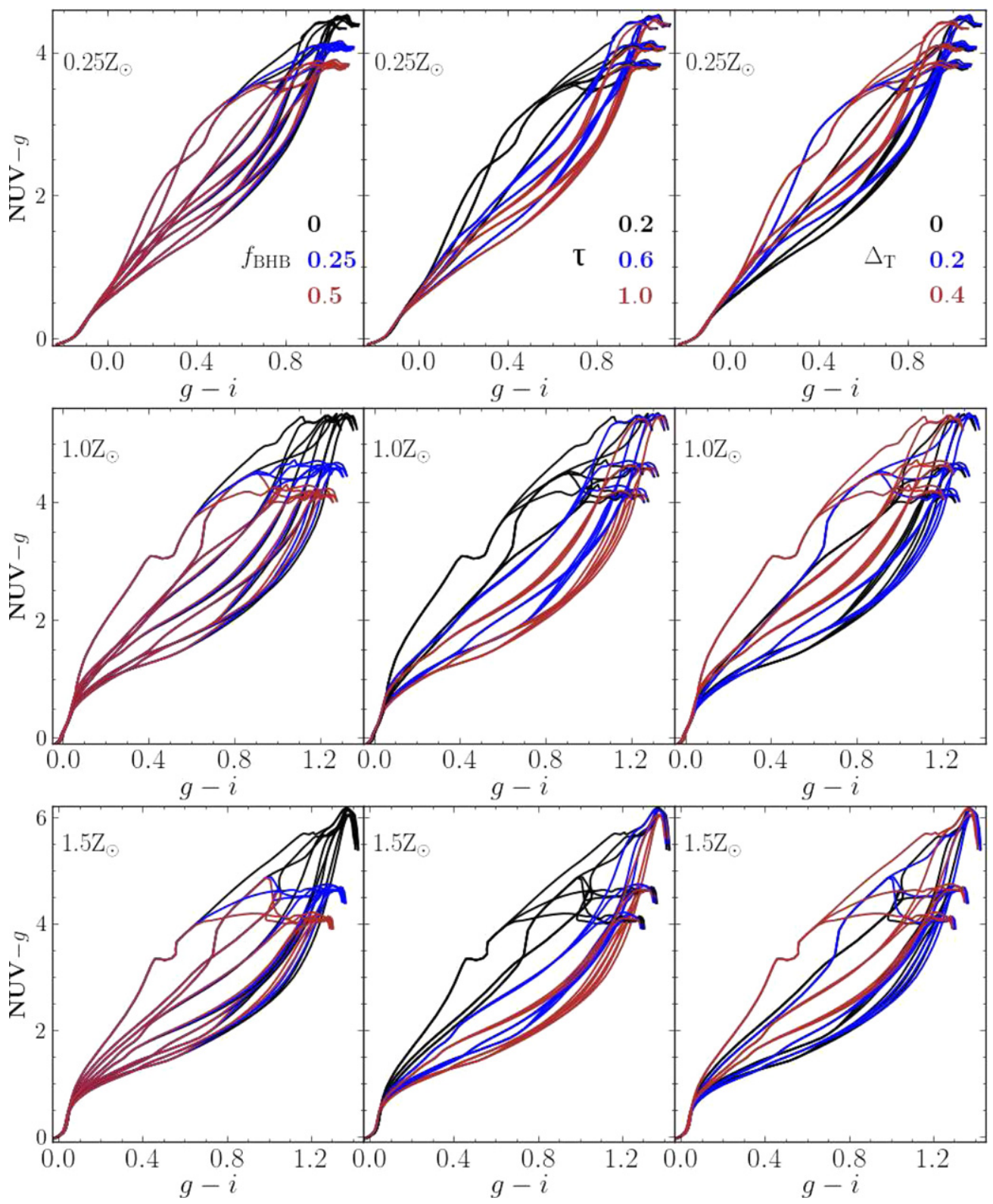

Figure 11. FSPS CSP templates depicting the evolution of NUV $-g$ vs. $g-i$ color-space from ages $0.03-14.1$ Gyr. See Figure 10 for a full description.

discussions on evolved stellar populations, and D. Stern for numerous insights into the discussion of galaxy evolution. We also thank R. Assef for his feedback on the analysis and early development of this project. This publication makes use of data products from the Wide-field Infrared Survey Explorer, which is a joint project of the University of California, Los Angeles, and the Jet Propulsion Laboratory/California Institute of Technology, funded by the National Aeronautics and Space Administration. The publication is based on observations made with the NASA Galaxy Evolution Explorer. GALEX is operated for NASA by the California Institute of Technology under NASA contract NAS5-98034. This publication makes use of data products from the Sloan Digital Sky Survey. Funding for the SDSS and SDSS-II has been provided by the Alfred P. Sloan Foundation, the Participating Institutions, the National Science Foundation, the U.S. Department of Energy, the National Aeronautics and Space Administration, the Japanese Monbukagakusho, the Max Planck Society, and the Higher Education Funding Council for England. The SDSS Web site is http://www.sdss.org/.
This publication makes use of data products from the Two Micron All Sky Survey, which is a joint project of the University of Massachusetts and the Infrared Processing and Analysis Center/California Institute of Technology, funded by the National Aeronautics and Space Administration and the National Science Foundation. This research has made use of the NASA/ IPAC Extragalactic Database (NED) which is operated by the Jet Propulsion Laboratory, California Institute of Technology, under contract with the National Aeronautics and Space Administration.

\section{APPENDIX}

\section{COLOR ANALYSIS OF UVX IN STELLAR POPULATIONS}

We used the FSPS photometry to determine the best approach for comparing UV-optical, optical-optical, optical-mid-IR, and UV-mid-IR colors. The analysis was carried out as described in Section 3. We briefly recap the CSP model parameters here. We combine the FSPS models with the Padova isochrones (Marigo 

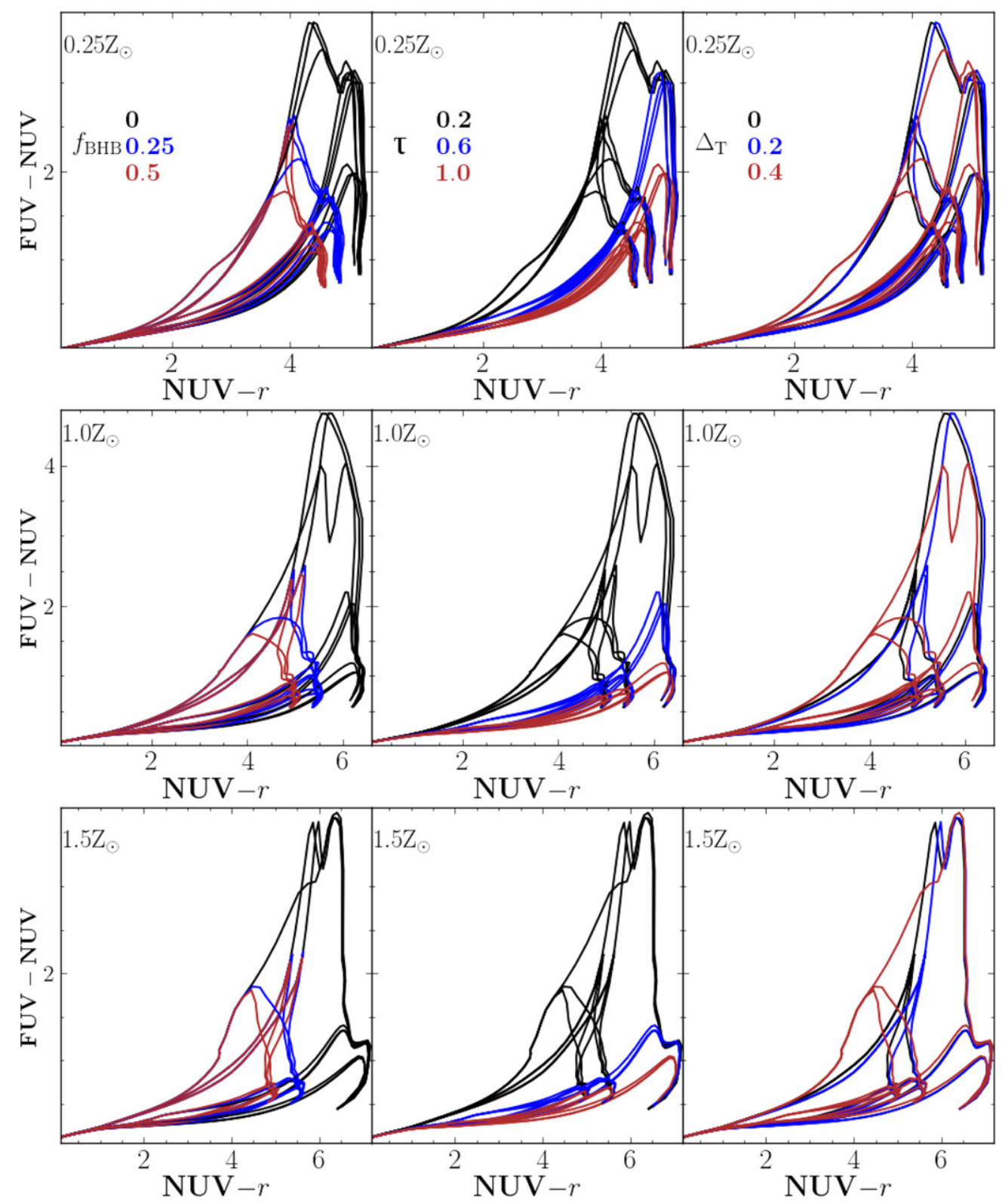

Figure 12. FSPS CSP templates depicting the evolution of FUV - NUV vs. NUV $-r$ color-space from ages $0.03-14.1$ Gyr. See Figure 10 for a full description.

\& Girardi 2007; Marigo et al. 2008) and limit the metallicities to $0.25,1$, and $1.5 Z_{\odot}$, ages between $30 \mathrm{Myr}$ and $14.1 \mathrm{Gyr}$ at $\Delta \log (t / \mathrm{yr})=0.025$ intervals, apply a Charlot \& Fall (2000) dust parameter of 0.3, and use the Chabrier (2003) IMF.

For BHBs, we assume that stars on the HB are allowed to become $\mathrm{BHB} / \mathrm{EHB}$ at ages $>2 \mathrm{Gyr}$, taking three different fractions of stars on the $\mathrm{HB}$ that are in the $\mathrm{BHB}$ (or EHB) phase: $0 \%, 25 \%$, and $50 \%$. To address the EHB phase, assuming these are BHBs with higher effective temperatures, we include temperature boosts $\Delta_{T}=0.2,0.4$ dex.

In Figures 10-13, we show how colors evolve with changes in the parameters, using NUV $-r / g-r$, NUV $-g / g-i$, FUV - NUV/NUV - $r$, FUV - NUV/NUV - [3.4]. Each panel from left to right shows the same FSPS tracks, but the lines are colored differently based on parameter values to emphasize the dependence of that parameter on the position in color-space (see labels in the top row). For example, the top row in Figure 10 shows (left) the BHB fractions of $0,0.25$, and 0.5 depicted in black, blue, and brown, respectively; (middle) the SFH $\tau=0.2$,
0.6, and 1 Gyr in black, blue, and brown, respectively; (right) the change in effective temperature $\Delta_{T}$ at $0,0.2$, and 0.4 dex in black, blue, and brown, respectively. The consecutive rows show how the FSPS tracks change with $Z$ as indicated in each panel.

Separation in color-space for each variable is not strong for $g-r, \mathrm{NUV}-g$, and $g-i$. All three have smaller ranges and smaller separations, leading to significant degeneracies. Concerning the former point, the color ranges in the panels in Figure 10 are very narrow, spanning 0.7 in optical, compared with the NUV $-r$ and NUV $-g(\sim 6)$. For the second point, the largest difference between the BHB and $\tau$ lines for $g-i$ and $g-r$ are $\lesssim 0.1$, and 0.3 , respectively. Separation in $\tau$ is reasonable, but separation in BHB fraction and temperature is relatively poor except at very old ages.

We extend our analysis to show the same tracks (with the same color-coding) in two examples of color-spaces that combine UV and mid-IR data; FUV - NUV/NUV - [3.4] and FUV - NUV/NUV $-r$ (Figure 11). These show a much wider 

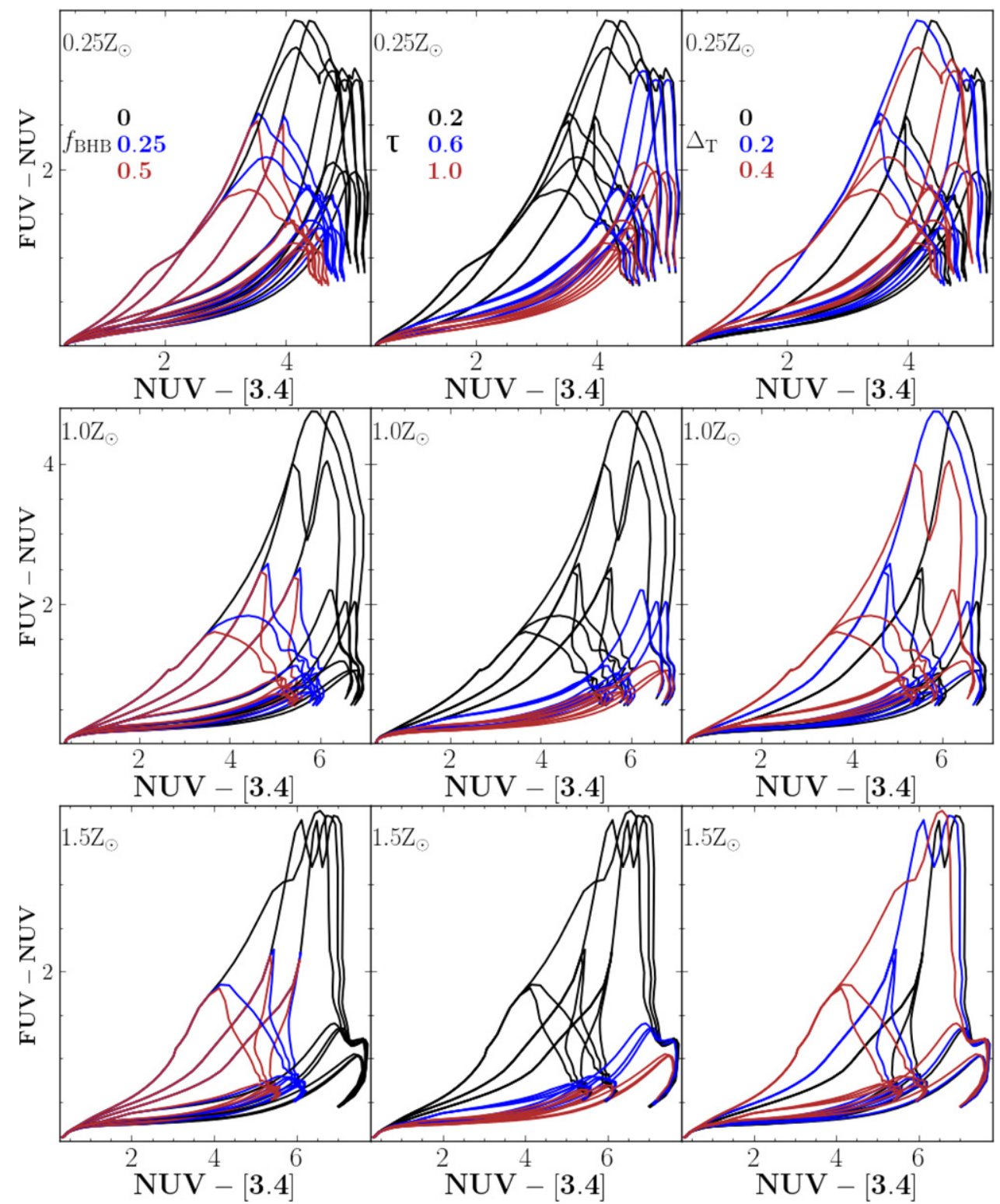

Figure 13. FSPS CSP templates depicting the evolution of FUV - NUV vs. NUV - [3.4] color-space with from ages 0.03-14.1 Gyr. See Figure 10 for a full description.

span in color on the $x$-axis by 5 mag, compared to the 0.7 mag range of the NUV $-r / g-r$ and NUV $-g / g-i$ plots (Figure 10 ).

Additionally, there is a much cleaner separation in color for $\tau$, and somewhat cleaner separations for both BHB and temperature. The GALEX-WISE colors provide an expanded range in color-space for BHB fraction color-cuts, especially at high metallicity, and there is a marginal improvement for changes in $\Delta_{T}$. For example, comparing the top left plots in Figures 10 and 11, the different BHB fractions are separated on average by $1 \mathrm{mag}$ in the GALEX-WISE, but only $0.2 \mathrm{mag}$ in the GALEX-SDSS. A further advantage of NUV - [3.4] colors is the near all-sky coverage of both datasets. Because of these advantages, we adopted the FUV - NUV/NUV - [3.4] and FUV - NUV/NUV $-r$ spaces as the primary color-space focus throughout the paper.

\section{REFERENCES}

Arnold, J. A., Romanowsky, A. J., Brodie, J. P., et al. 2011, ApJL, 736, L26 Atlee, D. W., Assef, R. J., \& Kochanek, C. S. 2009, ApJ, 694, 1539

Barnes, J. E., \& Hernquist, L. 1996, ApJ, 471, 115
Bell, E. F., McIntosh, D. H., Katz, N., \& Weinberg, M. D. 2003, ApJS, 149, 289 Brown, T. M., Ferguson, H. C., \& Davidsen, A. F. 1995, ApJL, 454, L15 Brown, T. M., Ferguson, H. C., Smith, E., et al. 2003, ApJL, 584, L69

Bureau, M., Jeong, H., Yi, S. K., et al. 2011, MNRAS, 414, 1887

Burstein, D., Bertola, F., Buson, L. M., Faber, S. M., \& Lauer, T. R. 1988, ApJ, 328,440

Carter, D., Pass, S., Kennedy, J., Karick, A. M., \& Smith, R. J. 2011, MNRAS, 414,3410

Chabrier, G. 2003, PASP, 115, 763

Charlot, S., \& Fall, S. M. 2000, ApJ, 539, 718

Code, A. D., \& Welch, G. A. 1979, ApJ, 228, 95

Conroy, C., \& Gunn, J. E. 2010, ApJ, 712, 833

Conroy, C., Gunn, J. E., \& White, M. 2009, ApJ, 699, 486

Cowie, L. L., Songaila, A., Hu, E. M., \& Cohen, J. G. 1996, AJ, 112, 839

Cox, T. J., Dutta, S. N., Di Matteo, T., et al. 2006, ApJ, 650, 791

Daddi, E., Renzini, A., Pirzkal, N., et al. 2005, ApJ, 626, 680

Damjanov, I., McCarthy, P. J., Abraham, R. G., et al. 2009, ApJ, 695, 101

De Lucia, G., Springel, V., White, S. D. M., Croton, D., \& Kauffmann, G. 2006, MNRAS, 366, 499

de Vaucouleurs, G., de Vaucouleurs, A., Corwin,, et al. 1991, Third Reference Catalogue of Bright Galaxies. (Berlin: Springer) (RC3)

de Zeeuw, P. T., Bureau, M., Emsellem, E., et al. 2002, MNRAS, 329, 513

Dorman, B., Rood, R. T., \& O'Connell, R. W. 1993, ApJ, 419, 596

Emsellem, E., Cappellari, M., Krajnović, D., et al. 2007, MNRAS, 379, 401

Ferguson, H. C., \& Davidsen, A. F. 1993, ApJ, 408, 92 
Forbes, D. A., Spitler, L. R., Strader, J., et al. 2011, MNRAS, 413, 2943

Gil de Paz, A., Boissier, S., Madore, B. F., et al. 2007, ApJS, 173, 185

Greggio, L., \& Renzini, A. 1990, ApJ, 364, 35

Han, Z., Podsiadlowski, P., \& Lynas-Gray, A. E. 2007, MNRAS, 380, 1098

Heber, U. 2009, ARA\&A, 47, 211

Jarrett, T. H., Chester, T., Cutri, R., Schneider, S. E., \& Huchra, J. P. 2003, AJ, 125,525

Jarrett, T. H., Masci, F., Tsai, C. W., et al. 2013, AJ, 145, 6

Jeong, H., Yi, S. K., Bureau, M., et al. 2012, MNRAS, 423, 1921

Kaluzny, J., \& Udalski, A. 1992, AcA, 42, 29

Kaviraj, S., Peirani, S., Khochfar, S., Silk, J., \& Kay, S. 2009, MNRAS, 394, 1713

Kaviraj, S., Schawinski, K., Devriendt, J. E. G., et al. 2007, ApJS, 173, 619

Kennicutt, R. C., Jr. 1998, ARA\&A, 36, 189

Kormendy, J., Fisher, D. B., Cornell, M. E., \& Bender, R. 2009, ApJS, 182, 216

Lee, H.-c., Yoon, S.-J., \& Lee, Y.-W. 2000, AJ, 120, 998

Lejeune, T., Cuisinier, F., \& Buser, R. 1997, A\&AS, 125, 229

Lejeune, T., Cuisinier, F., \& Buser, R. 1998, A\&AS, 130, 65

Liebert, J., Saffer, R. A., \& Green, E. M. 1994, AJ, 107, 1408

Marigo, P., \& Girardi, L. 2007, A\&A, 469, 239

Marigo, P., Girardi, L., Bressan, A., et al. 2008, A\&A, 482, 883

Martin, D. C., Fanson, J., Schiminovich, D., et al. 2005, ApJL, 619, L1

McDermid, R. M., Emsellem, E., Shapiro, K. L., et al. 2006, MNRAS, 373, 906

Neill, J. D., Sullivan, M., Gal-Yam, A., et al. 2011, ApJ, 727, 15

Neill, J. D., Sullivan, M., Howell, D. A., et al. 2009, ApJ, 707, 1449

Nelan, J. E., Smith, R. J., Hudson, M. J., et al. 2005, ApJ, 632, 137

Nelson, E. J., van Dokkum, P. G., Brammer, G., et al. 2012, ApJL, 747, L28
O’Connell, R. W. 1999, ARA\&A, 37, 603

O’Connell, R. W., Bohlin, R. C., Collins, N. R., et al. 1992, ApJL, 395, L45

Percival, S. M., \& Salaris, M. 2011, MNRAS, 412, 2445

Perrett, K. M., Bridges, T. J., Hanes, D. A., et al. 2002, AJ, 123, 2490

Pipino, A., \& Matteucci, F. 2004, MNRAS, 347, 968

Pipino, A., Matteucci, F., \& Chiappini, C. 2006, ApJ, 638, 739

Saito, Y., \& Iye, M. 2000, ApJL, 535, L95

Salim, S., Charlot, S., Rich, R. M., et al. 2005, ApJL, 619, L39

Sánchez-Blázquez, P., Gibson, B. K., Kawata, D., Cardiel, N., \& Balcells, M. 2009, MNRAS, 400, 1264

Sánchez-Blázquez, P., Gorgas, J., \& Cardiel, N. 2006, A\&A, 457, 823

Saracco, P., Gargiulo, A., \& Longhetti, M. 2012, MNRAS, 422, 3107

Sarzi, M., Bacon, R., Cappellari, M., et al. 2007, NewAR, 51, 18

Schawinski, K., Kaviraj, S., Khochfar, S., et al. 2007, ApJS, 173, 512

Schlegel, D. J., Finkbeiner, D. P., \& Davis, M. 1998, ApJ, 500, 525

Shapiro, K. L., Falcón-Barroso, J., van de Ven, G., et al. 2010, MNRAS, 402, 2140

Skrutskie, M. F., Cutri, R. M., Stiening, R., et al. 2006, AJ, 131, 1163

Trager, S. C., Faber, S. M., Worthey, G., \& González, J. J. 2000, AJ, 120, 165

Trager, S. C., Worthey, G., Faber, S. M., \& Dressler, A. 2005, MNRAS, 362, 2

Treu, T., Ellis, R. S., Liao, T. X., et al. 2005, ApJ, 633, 174

van Dokkum, P. G. 2005, AJ, 130, 2647

Wright, E. L., Eisenhardt, P. R. M., Mainzer, A. K., et al. 2010, AJ, 140, 1868

Yi, S., Demarque, P., \& Oemler, A., Jr. 1998, ApJ, 492, 480

Yi, S. K., Lee, J., Sheen, Y.-K., et al. 2011, ApJS, 195, 22

Yi, S. K., Yoon, S.-J., Kaviraj, S., et al. 2005, ApJL, 619, L111

York, D. G., Adelman, J., Anderson, J. E., Jr., et al. 2000, AJ, 120, 1579 